\title{
Biomimetic object impedance control for dual-arm cooperative 7-DOF manipulators
}

\author{
Yi Ren, Yechao Liu*, Minghe Jin, Hong Liu \\ State Key Laboratory of Robotics and System, Harbin Institute of Technology, 150001 Harbin, Heilongiang Province, China
}

\section{H I G H L I G H T S}

- A novel biomimetic object impedance controller is proposed.

- Object/internal impedance are simultaneously achieved in a unified architecture.

- Stiffness and damping of the object impedance can be adapted accordingly.

- Agilely dealing with unknown interactions for object/dual-arm system is enabled.

- Strict stability analysis with simulation and experiments are given.

\section{A R T I C L E I N F O}

\section{Article history:}

Received 5 April 2015

Received in revised form 28 August 2015

Accepted 21 September 2015

Available online 30 September 2015

\section{Keywords:}

Robotic control

Biomimetic object impedance

Cooperative humanoid manipulators

\begin{abstract}
A B S T R A C T
To mimic human behavior is an increasing trend for advanced robot control. In this paper, a novel biomimetic object impedance control strategy (BOI) is presented for dual-arm cooperative 7-DOF humanoid manipulators. A general impedance architecture comprising internal impedance control and object impedance control is adopted, which aims at conferring impedance behavior both in end-effector level and object level. Asymptotic stability and convergence of this controller are strictly derived from Lyapunov stability theory. Compared with conventional object impedance, the proposed controller can show different object impedance characteristics according to the external force applied on the object. It also can simultaneously minimize the energy cost of the adaptation process. Simulation and experimental results indicate that this controller exhibits explicit compliance behavior when the interaction with environment is weak and presents accumulation property of the stiffness and damping accordingly when the interaction is strong. This human-like characteristic enables the object/dual-arm system to deal with complex and unknown environmental disturbances.
\end{abstract}

(C) 2015 Elsevier B.V. All rights reserved.

\section{Introduction}

Dual-arm cooperative humanoid manipulators have significant advantages over other single manipulator for heavy load manipulation/transportation or dexterous assembly task as they can perform tasks in a human-like manner [1]. Although various controllers for cooperative manipulators have been proposed in recent years, stable execution of contact tasks with unknown environment using mechanical manipulators is still identified as one of the major challenges.

Pure position control may lead to many problems (such as the saturation of the actuators). The impedance control first proposed by Hogan $[2,3]$ provided a new way to solve the problem in-

\footnotetext{
* Corresponding author. Tel.: +86 15945185984.

E-mail addresses: yiporhit@163.com (Y. Ren), yechao_hitdlr@163.com (Y. Liu).
}

duced by contact force while simultaneously preserve motion stability during the contact between the object and the environment $[4,5]$. Even then, the adoption of impedance control in manipulators is much different. When dual-arm manipulators are employed to manipulate a common object, it needs to control both the relative motion of the manipulators and the internal stresses on the grasped object, thus the mappings between forces/velocities of each manipulator's end-effector and those of manipulated object need to be considered [6]. Lee et al. [7] proposed a relative impedance control strategy for dual-arm robots to perform asymmetric bimanual tasks based on relative Jacobian with time delay estimation (TDE) and ideal velocity feedback (IVF) techniques, this control strategy simplified the implementation compared to other traditional schemes. Zhu and Schutter [8] designed a novel controller for dual-arm system based on adaptive force/motion control.

Moreover, for cooperative manipulators interacting with the environment, many high performance results are available. 


\section{Nomenclature}

$\Sigma_{\text {World }}, \Sigma_{\boldsymbol{o}}$ World and object framework

$\Sigma_{R}, \Sigma_{L}$ Base framework of the two manipulators

$\Sigma_{1}, \Sigma_{2}$ Framework of the two end-effectors

$\boldsymbol{p}_{\boldsymbol{R}}, \boldsymbol{p}_{\mathbf{L}} \quad$ Translational vectors

$\boldsymbol{R}_{\boldsymbol{R}}, \boldsymbol{R}_{L}, \boldsymbol{R}_{\boldsymbol{o}}$ Orientation matrix of the right/left manipulator and the object

$\boldsymbol{F} \quad$ Contact force exerted by the two manipulators

$\boldsymbol{M}_{o}, \boldsymbol{G}_{0} \quad$ Object inertial matrix and gravity vector

$\boldsymbol{V}_{o}(\dot{\boldsymbol{y}}, \boldsymbol{y}) \dot{\boldsymbol{y}}$ Coriolis and centrifugal forces vector of the object

$\dot{\boldsymbol{y}}, \dot{\boldsymbol{x}} \quad$ Velocity vector of the object and the end-effectors

$\boldsymbol{y}_{d}, \boldsymbol{x}_{d} \quad$ Desired object and end-effector trajectories

$\boldsymbol{y}_{r}, \boldsymbol{x}_{r} \quad$ Reference trajectories of the object and endeffectors

$\boldsymbol{F}_{0} \quad$ Force/moment vector acting on object by the two manipulators

$\boldsymbol{F}_{\text {env }} \quad$ Wrench exerted by the environment on the object

$J_{0}^{T} \quad$ Grasp matrix

$\boldsymbol{M}(\boldsymbol{q}), \boldsymbol{G}(\boldsymbol{q})$ Manipulator inertial matrix and gravity vector

$\boldsymbol{V}(\dot{\boldsymbol{q}}, \boldsymbol{q}) \dot{\boldsymbol{q}}$ Coriolis and centrifugal forces vector of the manipulators

q Manipulator joint position vector

$\boldsymbol{\tau}, \boldsymbol{\tau}_{e} \quad$ Applied and mapping equivalent joint torques

$\boldsymbol{J}(\boldsymbol{q}), \boldsymbol{J}^{\dagger}(\boldsymbol{q})$ Analytical Jacobian of the redundant manipulators and its pseudo inverse

$\boldsymbol{F}_{E}, \boldsymbol{F}_{I} \quad$ Motion-inducing force and internal force

$\boldsymbol{M}_{\text {oI }}, \boldsymbol{D}_{\text {oI }}, \boldsymbol{K}_{\text {oI }}$ Inertial, damping and stiffness matrix of the object impedance

$\boldsymbol{M}_{e}, \boldsymbol{D}_{e}, \boldsymbol{K}_{e}$ Inertial, damping and stiffness matrix of the internal impedance

$\boldsymbol{s} \quad$ The combined error measure of the object

$\boldsymbol{Q}_{D}, \boldsymbol{Q}_{K} \quad$ Symmetric positive-definite gain matrix

$\boldsymbol{e}_{v}, \boldsymbol{e}_{p} \quad$ Task-space velocity and position error

Bonitz [9] successfully achieved the bounded internal forces by proposing an impedance control strategy, which controls the motion of the manipulated object and the internal forces. Based on previous work, Koga and Wimböck [10,11] proposed two different impedance laws for cooperative manipulators; both of them further put the interaction between the object and the environment into consideration. Schneider et al. [12] then extended the architecture of the impedance control for dual-arm cooperation creatively. They achieved the governing of the object impedance rather than just the impedance of manipulators' end-effectors. Since the estimation of the object's dynamics is inevitably noisy and inaccurate, the requirement of the object's dynamics limits extensive application of object impedance control. To avoid this limitation, Lian and Gueaieb [13-15] presented some enlightening algorithms based on intelligent control. Furthermore, Caccavale $[16,17]$ successfully combined the internal and external object impedance. His work simultaneously eliminated the dependence of object's dynamics by proper specification of some parameters. Some other researchers like Abdallah [18] and Moosavian [19] also presented various impedance laws (i.e. Multiple-impedance control) for cooperative manipulators to achieve both object and internal impedance.

However, to the authors' knowledge, in most of the previous works, the stiffness and damping of the object impedance are all fixed and cannot be adaptively modified according to the different interactions between the environment and the object. Few works integrated the adaptation law into the object impedance. Manipulators lacking adaptation capabilities are generally not able to deal with unknown interactions agilely. Besides, conventional method with large stiffness and damping will consume large amount of energy, which is not necessary especially when no environmental disturbance exists. Inspired by the works in [20,21] and then following the guidelines in [22-24], we proposed a whole control strategy with biomimetic object impedance for dual-arm system. This controller is expected to achieve the goal that the grasped object exhibits explicit compliance behavior when the interaction with environment is small and shows accumulation property of the stiffness and damping when it is large.

In addition, it needs to be mentioned that dual-arm system is different from multi-fingered system in some aspects. Unlike the object-level grasp controllers for robotic hands, multi-fingered hands are only capable of applying forces to the object while arms are capable of applying forces and moments. The serial of kinematic chain for dual-arm system is totally determined and the grasp allows bilateral force transmission [25,26], therefore the friction constrains existing in multi-fingered hands are negligible here.

This paper is organized as follows. The first part outlines an overall architecture for the control of dual-arm cooperative humanoid manipulators, which permits biomimetic object impedance control and simultaneously achieves the control of the internal force. This unified framework allows individual activation or deactivation of object and internal impedance, which may contribute to the simplification of the derivation of the adaptation law and stability analysis. This framework consists of two parts: the first part generates the Cartesian reference trajectory for the end-effectors, which is aiming at achieving impedance behavior both in object level and end-effector level. In addition, to deal with unknown interactions between the object and the environment, biomimetic object impedance strategy is designed. The resulting compliant motion is used to generate the reference trajectories for the object. Furthermore, to avoid large internal force and possible grasp failure, internal impedance between the end-effector's position/orientation displacements and the internal forces is designed to track the commanded internal forces, this is used to generate the reference motions for each end-effector [27]; The other part is a hierarchical position control with Cartesian position control as the primary loop and joint position control as the secondary loop respectively, which can ensure the two manipulators to achieve perfect tracking of the reference trajectory. Then the strict theoretical derivation of the proposed adaptive object impedance control is given. Finally, simulation of the proposed impedance control scheme is enforced. The results verify the effectiveness of the biomimetic object impedance control while building of large values of internal forces and grasp failure is avoided.

\section{Biomimetic adaption of object impedance control}

\subsection{Human behavior analysis of grasping an object}

When human arms grasp and manipulate an object, the operator sometimes needs to compensate for the forces applied on the object to avoid instability induced by the environmental interference. The intrinsic properties of muscles and reflex arcs, which refer to the muscle viscoelasticity and the stretch reflex, can collaboratively ensure a smooth and stabilized motion even with a reflection time delay of over $200 \mathrm{~ms}$ [28]. This mechanism, in essence, enables the human to adapt the damping and stiffness both in the arm impedance and the object impedance to compensate for the unknown dynamic environments.

Consider human using two arms to grasp an object cooperatively. When the object is free of interactions, human usually maintains a minimum stiffness and damping. This is significant to avoid unnecessary energy consumption. When the object is interfered 
by disturbance from the environment, two arms will move following the direction of external forces and simultaneously force the grasped object deviate along the same direction. Meanwhile, a restoring force in the opposite direction is generated. It is approximately proportional to the position error in this case [29]. When the external force and the restoring force achieve balance, the object reaches the equilibrium position. If the external force continues to increase, the increment of offset will decrease although the object will further deviate from the desired position. This means that the object grasped by human arms gets more and more difficult to be pushed forward, namely, the stiffness of the object responding to the external disturbance gradually increases. However, if the external force constantly changes, especially in its direction, such as swaying back and forth, the two arms will not completely follow the action of external force applied on the object, but become more viscous, this makes the restoring force respond to the variation of the external interference much faster and keeps the object around the desired position as close as possible, that is, the external damping of the object increases.

As is shown in the above analysis, when human grasps an object with his two arms, the object will exhibit a characteristic of impedance due to the action of the arms. The stiffness and damping of this impedance, which is known as object impedance, will adapt according to the environmental interference. The adaption of stiffness plays a major role when the disturbance is relatively stable, whereas the adaption of damping predominates when the disturbance changes dramatically.

Here we try to mimic this mechanism and propose a novel control strategy to endow the dual-arm cooperative 7-DOF humanoid manipulators an analogous ability.

\subsection{Dynamics and kinematics}

First of all, a basic assumption made here is that each arm is capable of imparting arbitrary force/moment to the object. In addition, it is also assumed that each end-effector of the dual-arm manipulator system grasps the object rigidly, their positions and orientations do not change relatively to the local coordinate of the object [30].

To simplify the derivation presented below, all the quantities written in bold in this paper means that they are vectors or matrixes.

Two 7-DOF manipulators tightly grasping a common object are shown in Fig. 1. For ease of analysis, a set of reference frames are defined as follows: $\boldsymbol{\Sigma}_{\text {World }}$ denotes the world frame. $\boldsymbol{\Sigma}_{R}$ and $\boldsymbol{\Sigma}_{\boldsymbol{L}}$ are the base frames of right and left manipulator. $\boldsymbol{\Sigma}_{\mathbf{1}}$ and $\boldsymbol{\Sigma}_{\mathbf{2}}$ are the coordinate frames attached to the two end-effectors, $\boldsymbol{p}_{R}$ and $\boldsymbol{p}_{\boldsymbol{L}}$ denote their $(3 \times 1)$ position vectors, $\boldsymbol{R}_{\boldsymbol{R}}$ and $\boldsymbol{R}_{\mathbf{L}}$ are the $(3 \times 3)$ matrixes characterizing their orientations, with respect to the world frame $\boldsymbol{\Sigma}_{\text {World }}$. Let $\boldsymbol{\Sigma}_{\boldsymbol{o}}$ be the coordinate frame attached to the object with its origin in the object's center of mass, $\boldsymbol{p}_{\boldsymbol{o}}$ denotes its $(3 \times 1)$ origin's position vector and $\boldsymbol{R}_{\boldsymbol{o}}$ is the $(3 \times 3)$ orientation matrix, both with respect to the world frame. For simplicity, $\boldsymbol{R}_{\boldsymbol{R}}, \boldsymbol{R}_{\boldsymbol{L}}$ and $\boldsymbol{R}_{\boldsymbol{o}}$ are chosen to coincide with each other.

\section{Dynamics}

Since each manipulator contacts with the object rigidly, thus may both exert a force/moment respectively. For each manipulator $(i=1,2)$, let $(6 \times 1)$ vector $\boldsymbol{f}_{i}=\left(\boldsymbol{f}_{p i}^{T} \boldsymbol{n}_{\tau i}^{T}\right)^{T}$ denotes the wrench exerted by the manipulators on the object at the equivalent contact points, where $\boldsymbol{f}_{p i}$ is the $(3 \times 1)$ vector that expresses the force and $\boldsymbol{n}_{\tau i}$ is the $(3 \times 1)$ vector that denotes the moment. A fixed point in each of the two contact zones on the object is chosen as the corresponding equivalent contact point for simplifying mechanical analysis. Only by multiplying the measured force by a certain transformation matrix can we get the force vector $\boldsymbol{f}_{i}$. Here we use $(12 \times 1)$ vector $\boldsymbol{F}=\left[\begin{array}{ll}\boldsymbol{f}_{1}^{T} & \boldsymbol{f}_{2}^{T}\end{array}\right]^{T}$ to express the contact force.

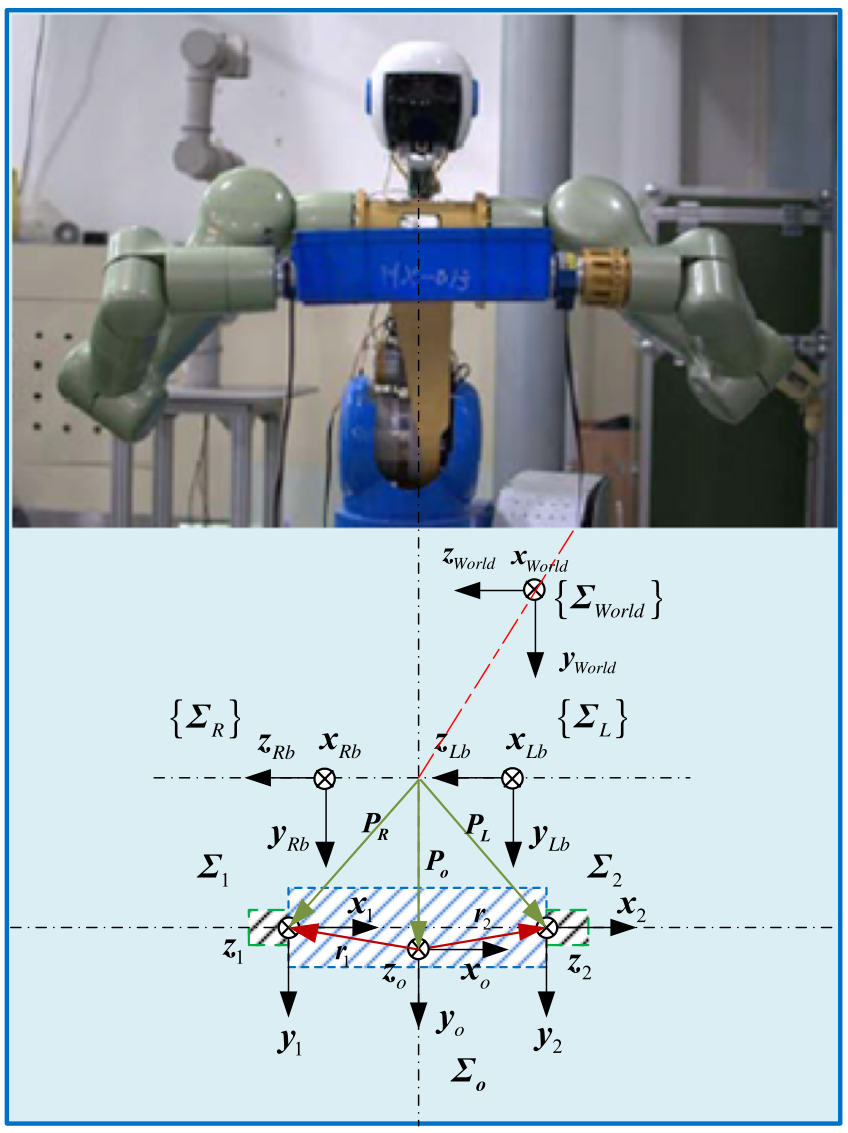

Fig. 1. The dual-arm system named HIT-Robonaut and the coordinate system when grasping an object.

The dynamics of the object can be described with respect to the world frame by the following equation in compact form:

$\boldsymbol{M}_{0} \ddot{\boldsymbol{y}}+\boldsymbol{V}_{o}(\dot{\boldsymbol{y}}, \boldsymbol{y}) \dot{\boldsymbol{y}}+\boldsymbol{G}_{o}=\boldsymbol{F}_{0}+\boldsymbol{F}_{\text {env }}$

where $\boldsymbol{M}_{o}$ consists of the object's mass matrix and inertia tensor, it depends not only on the relative position between the object's center of mass and the origin of the world frame $\boldsymbol{\Sigma}_{\text {World }}$, but also on the orientation of the frame $\boldsymbol{\Sigma}_{0}$, the term $\boldsymbol{V}_{o}(\dot{\boldsymbol{y}}, \boldsymbol{y}) \dot{\boldsymbol{y}}$ denotes the vector of centrifugal and Coriolis force and $\dot{\boldsymbol{y}}$ is the $(6 \times 1)$ object velocity vector referred to $\boldsymbol{\Sigma}_{\text {World }} . \boldsymbol{G}_{0}$ represents the $(6 \times 1)$ vector of gravitational forces, $\boldsymbol{F}_{e n v}$ is the $(6 \times 1)$ vector expressing the wrench exerted by the environment on the object. $\boldsymbol{F}_{0}$ is the $(6 \times 1)$ vector of force and moment acting on object by the two manipulators.

$\boldsymbol{F}_{o}=\boldsymbol{J}_{0}^{T} \boldsymbol{F}=\left[\begin{array}{ll}\boldsymbol{J}_{01}^{T} & \boldsymbol{J}_{02}^{T}\end{array}\right] \boldsymbol{F}$

where $\boldsymbol{J}_{0}^{T}$ is the $(6 \times 12)$ matrix known as grasp matrix.

$\boldsymbol{J}_{o i}^{T}=\left[\begin{array}{cc}\mathbf{I}_{3} & \mathbf{O}_{3} \\ \boldsymbol{P}_{i} & \mathbf{I}_{3}\end{array}\right]$

$\boldsymbol{P}_{i}=\left[\begin{array}{ccc}0 & p_{i z} & -p_{i y} \\ -p_{i z} & 0 & p_{i x} \\ p_{i y} & -p_{i x} & 0\end{array}\right]$.

The $(3 \times 1)$ vector $\boldsymbol{r}_{i}=\left[\begin{array}{lll}p_{i x} & p_{i y} & p_{i z}\end{array}\right](i=1,2)$ expresses the position vector from the object's center of mass to the contact point between the end-effector and object, which is relative to the object's local framework.

The dynamic model of the two manipulators can be depicted as:

$\boldsymbol{M}(\boldsymbol{q}) \ddot{\boldsymbol{q}}+V(\dot{\boldsymbol{q}}, \boldsymbol{q}) \dot{\boldsymbol{q}}+\boldsymbol{G}(\boldsymbol{q})=\boldsymbol{\tau}+\boldsymbol{\tau}_{\boldsymbol{e}}$ 


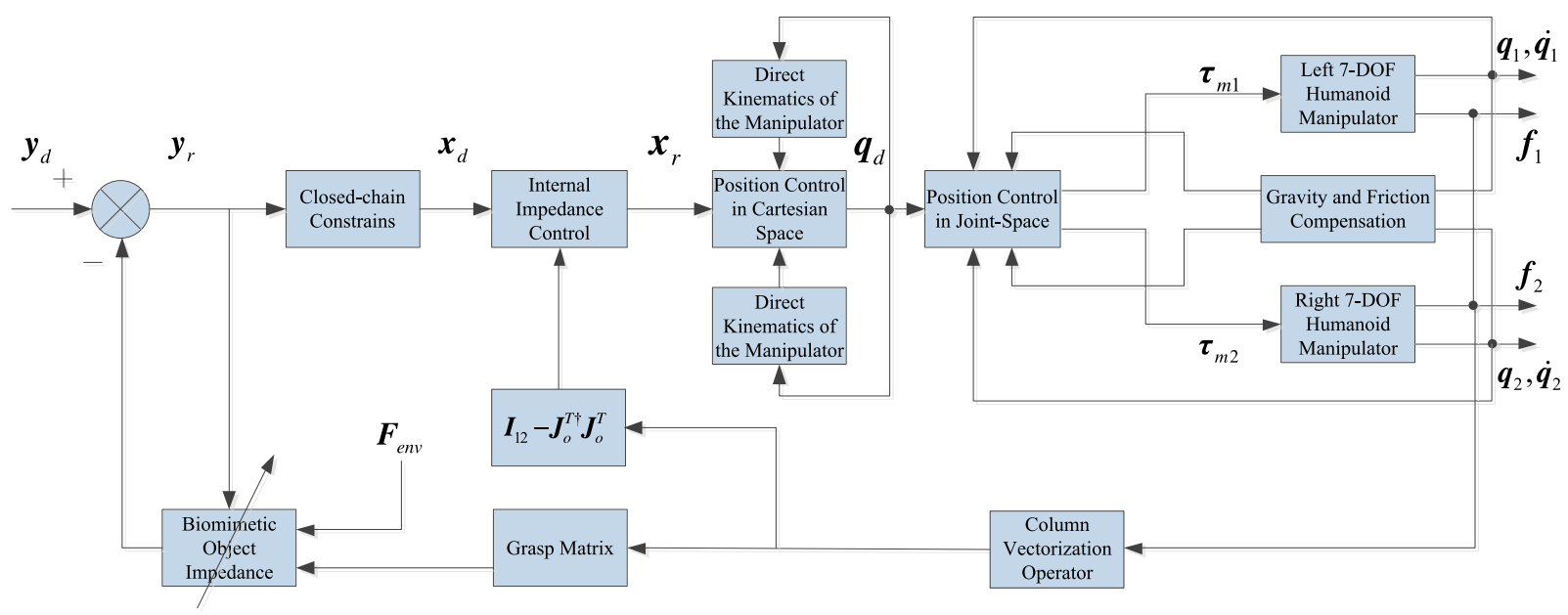

Fig. 2. Block diagram of the impedance control architecture.

where $\boldsymbol{M}(\boldsymbol{q})=\operatorname{diag}\left(\boldsymbol{M}_{1}\left(\boldsymbol{q}_{1}\right), \boldsymbol{M}_{2}\left(\boldsymbol{q}_{2}\right)\right) \in R^{14 \times 14}, \boldsymbol{M}_{i}\left(\boldsymbol{q}_{i}\right) \in R^{7 \times 7}$ denotes the symmetric positive definite inertial matrix of the $i$ th manipulator. $\boldsymbol{q}=\left(\boldsymbol{q}_{1}^{T}, \boldsymbol{q}_{2}^{T}\right)^{T} \in R^{14 \times 1}, \boldsymbol{q}_{i} \in R^{7 \times 1}$ denotes the vector of joint angles of each manipulator. $\boldsymbol{V}(\dot{\boldsymbol{q}}, \boldsymbol{q}) \dot{\boldsymbol{q}}=\operatorname{diag}\left(\boldsymbol{V}_{1} \dot{\boldsymbol{q}}_{1}, \boldsymbol{V}_{2} \dot{\boldsymbol{q}}_{2}\right)$ is the $(14 \times 1)$ vector of Coriolis and centrifugal forces, $\boldsymbol{\tau}=$ $\left(\boldsymbol{\tau}_{1}^{T}, \boldsymbol{\tau}_{2}^{T}\right)^{T} \in R^{14 \times 1}$ is the vector of applied joint torques and $\tau_{\boldsymbol{e}}=$ $\left(\boldsymbol{\tau}_{e 1}^{T}, \boldsymbol{\tau}_{e 2}^{T}\right)^{T} \in R^{14 \times 1}$ is the mapping equivalent joint torques from Cartesian space. $\boldsymbol{G}(\boldsymbol{q})=\operatorname{diag}\left(\boldsymbol{G}_{1}\left(\boldsymbol{q}_{1}\right), \boldsymbol{G}_{2}\left(\boldsymbol{q}_{2}\right)\right) \in R^{14 \times 1}$ represents the gravity term. $\boldsymbol{V}_{i} \dot{\boldsymbol{q}}_{i} \in R^{7 \times 1}, \boldsymbol{G}_{i}\left(\boldsymbol{q}_{i}\right) \in R^{7 \times 1}, \boldsymbol{\tau}_{i} \in R^{7 \times 1}$ and $\boldsymbol{\tau}_{\boldsymbol{e} i} \in$ $R^{7 \times 1}$ denote their counterparts of each manipulator respectively.

\section{Kinematics:}

According to the definition of the grasp matrix, the following mapping equation can be obtained:

$\dot{\boldsymbol{x}}=\left[\begin{array}{c}\dot{\boldsymbol{x}}_{1} \\ \dot{\boldsymbol{x}}_{2}\end{array}\right]=\boldsymbol{J}_{0} \dot{\boldsymbol{y}}=\left[\begin{array}{ll}\boldsymbol{I}_{3} & \boldsymbol{p}_{R} \\ \mathbf{0}_{3} & \boldsymbol{I}_{3} \\ \boldsymbol{I}_{3} & \boldsymbol{p}_{L} \\ \mathbf{0}_{3} & \boldsymbol{I}_{3}\end{array}\right] \dot{\boldsymbol{y}}$

where $\dot{\boldsymbol{x}}_{i}$ denotes the linear and angular velocity of the $i$ th endeffector referred to the base frame, it is a $(6 \times 1)$ vector.

The mapping from Cartesian space to joint space is described by:

$\dot{\boldsymbol{x}}=\boldsymbol{J}(\boldsymbol{q}) \dot{\boldsymbol{q}}$

$\boldsymbol{J}(\boldsymbol{q})=\left[\begin{array}{cc}\boldsymbol{J}_{1}\left(\boldsymbol{q}_{1}\right) & \mathbf{0}_{6 \times 7} \\ \mathbf{0}_{6 \times 7} & \boldsymbol{J}_{2}\left(\boldsymbol{q}_{2}\right)\end{array}\right]$

where $\boldsymbol{J}_{i}\left(\boldsymbol{q}_{i}\right)$ is a $(6 \times 7)$ matrix expressing the analytical Jacobian matrix of the manipulator for $i=1,2$. It should be noted that the vector of equivalent joint torques satisfies the equation:

$\tau_{e}=-J^{T}(q) F$.

\subsection{Decomposition of external force and internal force}

To achieve impedance behavior when the object interacts with the environment and the bounded internal forces, we need to simultaneously implement the object impedance control and internal impedance control. For this purpose, similar impedance control architecture [16] with some proper modifications can be adopted.

Since we put our emphasis on the implementation of biomimetic adaption of object impedance, the object and internal force should be decoupled first.

As depicted in [31], the force $\boldsymbol{F}$ applied by the manipulators can be decomposed into motion-inducing and internal force. Motioninducing force, expressed by a $(6 \times 1)$ vector $\boldsymbol{F}_{E}$, represents the force exerted by the end-effector that may balance the object's dynamics and the wrench exerted by the environment on the object. As well we use the $(6 \times 1)$ vector $\boldsymbol{F}_{I}$ to denote the internal force. The internal force that consists of compressive or tensile force and torque do not contribute to the motion of the object. Then we can get:

$\boldsymbol{F}=\boldsymbol{F}_{E}+\boldsymbol{F}_{I}$.

According to its definition, the internal force do not contribute to the actual force on the object, thus spanning the null space of $\boldsymbol{J}_{0}^{T}$, then the internal force and the motion-inducing force can be given by the following two equations:

$\boldsymbol{F}_{E}=\left[\begin{array}{ll}\boldsymbol{f}_{1 E} & \boldsymbol{f}_{2 E}\end{array}\right]^{T}=\boldsymbol{J}_{o}^{T \dagger} \boldsymbol{J}_{0}^{T} \boldsymbol{F}$

$\boldsymbol{F}_{I}=\left[\begin{array}{ll}\boldsymbol{f}_{1 I} & \boldsymbol{f}_{2 I}\end{array}\right]^{T}=\left(\boldsymbol{I}_{12}-\boldsymbol{J}_{o}^{T \dagger} \boldsymbol{J}_{0}^{T}\right) \boldsymbol{F}$

where $\boldsymbol{J}_{0}^{T \dagger}$ is a generalized inverse of the grasp matrix $\boldsymbol{J}_{0}^{T}$.

Since $\boldsymbol{J}_{o}^{T \dagger}=A \boldsymbol{J}_{o}\left(\boldsymbol{J}_{0}^{T} A \boldsymbol{J}_{o}\right)^{-1}$ and $A \in R^{6 \times 6}$ are positive definite matrixes, then different $\boldsymbol{A}$ may induce different solutions to the above equation. According to [30], by choosing $\boldsymbol{J}_{o}^{T \dagger}$ as Eq. (12), we can avoid inherent squeezing effect in motion-inducing force:

$\boldsymbol{J}_{0}^{T \dagger}=\frac{1}{2}\left[\begin{array}{cc}\boldsymbol{I}_{3} & \mathbf{0}_{3} \\ -\boldsymbol{P}_{1} & \boldsymbol{I}_{3} \\ \boldsymbol{I}_{3} & \mathbf{0}_{3} \\ -\boldsymbol{P}_{2} & \boldsymbol{I}_{3}\end{array}\right]$

$\boldsymbol{I}_{12}-\boldsymbol{J}_{0}^{T \dagger} \boldsymbol{J}_{0}^{T}=\frac{1}{2}\left[\begin{array}{cccc}\mathbf{0}_{6} & & -\boldsymbol{I}_{3} & \boldsymbol{0}_{3} \\ -\boldsymbol{I}_{3} & \mathbf{0}_{3} & \boldsymbol{P}_{2} & -\boldsymbol{I}_{3} \\ \boldsymbol{P}_{2}-\boldsymbol{P}_{1} & -\boldsymbol{I}_{3} & \mathbf{0}_{6} & \end{array}\right]$.

As presented above, since the force $\boldsymbol{F}$ can be decomposed into two parts, the internal force lies in the null space of $\boldsymbol{J}_{0}^{T}$, then external and internal forces can be decoupled.

\subsection{Impedance control architecture}

In this part, the impedance control scheme can be pursued, as in paper [16], and would be extended with proper modification according to the requirement of object impedance adaptation. The whole control strategy is depicted in Fig. 2.

Definitely, to implement the impedance task, we should assume that the inner position tracking control is accurate enough. Here we use hierarchical closed-loop control framework, Cartesian position control as the primary loop policy and joint position control as the secondary loop policy respectively. This framework can 
ensure high coincidence of the reference and the actual trajectory if the concerned parameters meet the corresponding stability requirements.

To achieve geometrically consistent stiffness, here we adopt Six-DOF Impedance, a generalized mechanical impedance encompassing both translational and rotational displacements first proposed in [32] and further extended in [27].

Then the object impedance equation and the internal impedance equation are given as follows:

$$
\begin{aligned}
& \boldsymbol{M}_{o I} \Delta \ddot{\boldsymbol{y}}+\boldsymbol{D}_{o I} \Delta \dot{\boldsymbol{y}}+\boldsymbol{K}_{o I} \Delta \boldsymbol{y}=-\boldsymbol{F}_{e n v} \\
& \boldsymbol{M}_{e} \Delta \ddot{\boldsymbol{x}}+\boldsymbol{D}_{e} \Delta \dot{\boldsymbol{x}}+\boldsymbol{K}_{e} \Delta \boldsymbol{x}=\Delta \boldsymbol{F}_{I} \\
& \Delta \boldsymbol{F}_{I}=\boldsymbol{F}_{I d}-\boldsymbol{F}_{I} \\
& \Delta \boldsymbol{y}=\boldsymbol{y}_{d}-\boldsymbol{y}_{r} \\
& \Delta \boldsymbol{x}=\boldsymbol{x}_{d}-\boldsymbol{x}_{r}
\end{aligned}
$$

where $\boldsymbol{y}_{d}$ and $\boldsymbol{x}_{d}$ are vectors denoting the desired object trajectories and desired end-effector trajectories for each manipulators, $\boldsymbol{y}_{r}$ and $\boldsymbol{x}_{r}$ are corresponding reference trajectories. $\boldsymbol{M}_{e}, \boldsymbol{D}_{e}$ and $\boldsymbol{K}_{e}$ are $(12 \times 12)$ symmetric positive-definite matrixes that denote the mass, damping and stiffness matrixes for internal impedance, respectively. $\boldsymbol{M}_{O I}, \boldsymbol{D}_{O I}$ and $\boldsymbol{K}_{O I}$ are $(6 \times 6)$ symmetric positivedefinite matrixes for object impedance, their adaptation law will be described in the next part. $\boldsymbol{F}_{I d}$ denotes the desired internal force which can avoid both the damage of the object and possible grasp failure. Different from the internal impedance as in [16], a commanded internal contact force can be specified here.

As to the object impedance described by (14), the direct measurement of $\boldsymbol{F}_{\text {env }}$, namely, the force exerted by the environment on the object, is not available, so (14) needs to be transformed. Only by folding the object dynamics (1) into Eq. (14) can we get:

$\boldsymbol{M}_{O I} \Delta \ddot{\boldsymbol{y}}+\boldsymbol{D}_{O I} \Delta \dot{\boldsymbol{y}}+\boldsymbol{K}_{O I} \Delta \boldsymbol{y}=-\boldsymbol{M}_{o} \ddot{\boldsymbol{y}}-\boldsymbol{V}_{o}(\dot{\boldsymbol{y}}, \boldsymbol{y}) \dot{\boldsymbol{y}}-\boldsymbol{G}_{0}+\boldsymbol{F}_{0}$.

In the above expression, it is not easy to get the accurate value of the object acceleration $\ddot{\boldsymbol{y}}$. For the high tracking accuracy of the inner position controller and the small error $\Delta \boldsymbol{x}$, a reasonable assumption can be made that frame $\Sigma_{0}$ coincides with the reference object frame $\Sigma_{\text {or }}$ (a virtual frame which depicts the reference trajectory of the object). Accordingly, the object's actual acceleration equals the object reference acceleration. Since $\boldsymbol{F}_{o}$ can be readily computed by (2), then $\boldsymbol{y}_{r}$ can be obtained by solving (19). Via using (15), the corresponding Cartesian reference trajectories $\boldsymbol{x}_{\mathrm{r}}$ can be readily computed.

To gain perfect tracking of the Cartesian reference position, here we adopt a Cartesian closed-loop algorithm [33] and resolve the redundancy of the 7-DOF manipulators at acceleration level [34]. The desired joint trajectories can be described as:

$$
\begin{aligned}
& \ddot{\boldsymbol{q}}_{d}=\boldsymbol{J}^{\dagger}(\boldsymbol{q})\left(\ddot{\boldsymbol{x}}_{r}+\boldsymbol{K}_{v x} \boldsymbol{e}_{v}+\boldsymbol{K}_{p x} \boldsymbol{e}_{p}-\dot{J}(\boldsymbol{q}) \dot{\boldsymbol{q}}_{d}\right)+\ddot{\boldsymbol{q}}_{N} \\
& \boldsymbol{e}_{v}=\left(\dot{\boldsymbol{x}}_{r}-\dot{\boldsymbol{x}}_{a}\right) \\
& \boldsymbol{e}_{p}=\left(\boldsymbol{x}_{r}-\boldsymbol{x}_{a}\right) \\
& \dot{\boldsymbol{x}}_{a}=\boldsymbol{J}(\boldsymbol{q}) \dot{\boldsymbol{q}}_{d} \\
& \boldsymbol{J}^{\dagger}(\boldsymbol{q})=\boldsymbol{J}^{\boldsymbol{T}}(\boldsymbol{q})\left(\boldsymbol{J}(\boldsymbol{q}) \boldsymbol{J}^{\boldsymbol{T}}(\boldsymbol{q})\right)^{-1}
\end{aligned}
$$

where $\ddot{\boldsymbol{q}}_{d}=\left[\begin{array}{ll}\ddot{\boldsymbol{q}}_{d 1}^{T} & \ddot{\boldsymbol{q}}_{d 2}^{T}\end{array}\right]^{T}$ is the $(14 \times 1)$ vector of joint desired accelerations for each manipulator. Kinematic singularity will be not considered here. $\boldsymbol{K}_{v x}$ and $\boldsymbol{K}_{p x}$ are $(12 \times 12)$ symmetric positivedefinite matrixes, which can ensure asymptotic stability of (20). $\boldsymbol{J}^{\dagger}(\boldsymbol{q})$ is the pseudo inverse of $\boldsymbol{J}(\boldsymbol{q})$; $\ddot{\boldsymbol{q}}_{N}$ is a $(14 \times 1)$ vector denoting the null-space joint accelerations, which can be used and controlled for null-space optimization task. By solving (19), (15), (20), the joint desired trajectories $\boldsymbol{q}_{d}$ can be obtained.

Then using a PD with Gravity and Friction compensation control law, the commanded torques of the motor can be given as Eq. (22).

$\boldsymbol{\tau}=\boldsymbol{K}_{p q} \boldsymbol{e}_{q}+\boldsymbol{K}_{v q} \dot{\boldsymbol{e}}_{q}+\boldsymbol{G}(\boldsymbol{q})+\boldsymbol{F}_{f}(\boldsymbol{q}, \dot{\boldsymbol{q}})$

$\boldsymbol{e}_{q}=\boldsymbol{q}_{d}-\boldsymbol{q}$ where $\boldsymbol{K}_{v q}$ and $\boldsymbol{K}_{p q}$ are $(14 \times 14)$ symmetric positive-definite gain matrixes, $\boldsymbol{G}(\boldsymbol{q})$ and $\boldsymbol{F}_{f}(\boldsymbol{q}, \dot{\boldsymbol{q}})$ are $(14 \times 1)$ vectors expressing the gravitational forces and friction forces, they are given by model-based computation and calibration experiment with proper modeling respectively. Then the closed-loop control in joint space can be achieved.

Remarkably, the adoption of PD with Gravity and Friction compensation controllers here is much more appropriate than some other dynamical model based or intelligent controllers due to unknown uncertainties and model parameter errors. To enhance the performance of the joint controller, gravity compensation are needed and the essential parameters of the two manipulators are obtained by their Pro/Engineer models respectively. The Stribeck static friction model used here is a tradeoff between computation cost and locating/tracking accuracy. Compared with the usual Coulomb-viscous friction model, it can reflect the nature of friction better. Also, this model has fewer parameters needed to be identified than other dynamic friction models. Since this model can compensate more than $90 \%$ of the actual friction, therefore sufficiently meets the requirements of locating/tracking accuracy in joint space.

\section{Notation:}

To implement the proposed control scheme, a set of signals that we cannot directly obtain by existing sensors is needed. The algorithms to obtain these signals are as follows:

Object position: The position of each modular joint can be measured by the integrated joint position sensor. With forward kinematics of the manipulators (defined as $\boldsymbol{x}=\phi_{m}(\boldsymbol{q})$ ) and closedchain constrains (defined as $\boldsymbol{y}=\phi_{G}(\boldsymbol{x})$ ) between the manipulators and the object [35], the following relation can be obtained:

$\boldsymbol{y}=\phi_{G}\left(\phi_{m}(\boldsymbol{q})\right)$

Velocity: Since the high-gain observer can estimate the derivative of the output signals and effectively suppress the disturbance brought by white noise simultaneously, and the whole process is independent of the mathematical model, here we use this method to observe the joint velocity. To eliminate the peak phenomenon existed in high-gain observer, original mathematical description should be modified. The transfer function defining the high-gain observer is given by

$\frac{\hat{\dot{q}}_{i j}}{q_{i j}}=G(s)=\frac{\varepsilon s}{\frac{\varepsilon^{3} s^{2}}{\alpha_{2}}+\frac{\alpha_{1} \varepsilon^{2} s}{\alpha_{2}}+\varepsilon}$

where $q_{i j}$ denotes the joint angle of the joints for $j=1,2 \ldots 7$ in manipulators for $i=1,2$, which is measured by the encoder integrated in each joint, $\hat{\dot{q}}_{i j}$ is the estimated value of the corresponding joint velocity. $\varepsilon$ is a small positive parameter to be specified, the positive constants $\alpha_{1}$ and $\alpha_{2}$ are chosen to satisfy the Hurwitz rules. These constrains can ensure the convergence of the estimated joint velocity [36].

Force: the terms of $\boldsymbol{F}_{0}$ and $\boldsymbol{F}_{I}$ in Eqs. (19) and (16) can be readily computed by Eqs. (2) and (11) respectively, only end-effector forces and moments need to be measured.

Also, current loop is introduced into motor control to achieve the approximately linear relationship between motor torque and control current, thus, relatively accurate output torque can be controlled independently.

\subsection{Biomimetic object impedance}

As to function (14) and (19), we need to avoid arbitrary assignment of the mass and inertia matrix since the accurate object acceleration is not available.

$\boldsymbol{M}_{o I}=\boldsymbol{K} \mathbf{M}_{o}$ 
where $\boldsymbol{K}$ is a diagonal gain matrix whose eigenvalues are all greater than or equal to 1 . This assignment can contribute to the compensation of the object dynamics in (19). Furthermore, choosing $\boldsymbol{K}=\mathbf{I}_{6}$ will avoid large modification of the system characteristics.

$\boldsymbol{M}_{O I}=\boldsymbol{M}_{0}$.

The stiffness and damping matrixes are respectively adapted according to:

$$
\begin{aligned}
\Delta \boldsymbol{D}_{O I} & =\boldsymbol{D}_{O I}(t)-\boldsymbol{D}_{O I}(t-T) \\
& =-\boldsymbol{Q}_{D} \cdot\left(\gamma \boldsymbol{D}_{o I}-\boldsymbol{s} \cdot \Delta \dot{\boldsymbol{y}}^{T}\right) \\
\Delta \boldsymbol{K}_{O I} & =\boldsymbol{K}_{O I}(t)-\boldsymbol{K}_{o I}(t-T) \\
& =-\boldsymbol{Q}_{K} \cdot\left(\gamma \boldsymbol{K}_{o I}-\boldsymbol{s} \cdot \Delta \boldsymbol{y}^{T}\right)
\end{aligned}
$$

$s=\Delta \dot{\boldsymbol{y}}+\boldsymbol{\Lambda} \Delta \boldsymbol{y}$

where $\boldsymbol{Q}_{D}$ and $\boldsymbol{Q}_{K}$ are symmetric positive-definite gain matrixes corresponding to the adaptation law of $\boldsymbol{K}_{O I}$ and $\boldsymbol{D}_{O I}$ respectively. $T$ is the sampling time. $\boldsymbol{\Lambda}$ is the positive-definite diagonal matrix, $\boldsymbol{s}$ denotes the combined error measure. With Barbalat's lemma, one can easily know the convergence of $\boldsymbol{s}$ to zero implies that of the error $\Delta \boldsymbol{y}$ and its time derivative $\Delta \dot{\boldsymbol{y}}$.

Eq. (28) can be divided into two terms. The first term, $-\boldsymbol{Q}_{D} \cdot \gamma \cdot \boldsymbol{D}_{o I}$ (or $-\boldsymbol{Q}_{K} \cdot \gamma \cdot \boldsymbol{K}_{o I}$ ), plays the role to limit the relative maximums of the damping (or stiffness), the second term, $\boldsymbol{Q}_{D} \cdot \boldsymbol{s} \cdot \Delta \dot{\boldsymbol{y}}^{T}$ (or $\boldsymbol{Q}_{K} \cdot \boldsymbol{s} \cdot \Delta \boldsymbol{y}^{T}$ ), ensures the damping (or stiffness) adapting with the velocity error (or position error) and changing toward the direction in which deviation of the object decreases. This design enables the object/dual-arm system behaves like human beings as aforementioned.

To simplify the derivation below, two following error equations are defined:

$\tilde{\boldsymbol{D}}_{o I}=\hat{\boldsymbol{D}}_{o I}-\boldsymbol{D}_{o I}$

$\tilde{\boldsymbol{K}}_{o I}=\hat{\boldsymbol{K}}_{o I}-\boldsymbol{K}_{o I}$

where $\hat{\boldsymbol{D}}_{o I}$ and $\hat{\boldsymbol{K}}_{o I}$ are the minimal desired damping and stiffness that can maintain stability and reduce the deviation between the desired trajectory and reference trajectory.

\section{Stability and convergence analysis}

Stability and convergence of the whole control system, described by three closed-loop Eqs. (14), (15) and (20) with two adaption laws (28), is investigated below.

Cartesian position error is defined as $\Delta \tilde{\boldsymbol{x}}=\boldsymbol{x}_{r}-\boldsymbol{x}_{a}$, then a candidate Lyapunov function for the inner-loop position control is given by:

$V_{1}=\frac{1}{2} \Delta \dot{\tilde{\boldsymbol{x}}}^{T} \Delta \dot{\tilde{\boldsymbol{x}}}+\frac{1}{2} \Delta \tilde{\boldsymbol{x}}^{T} \boldsymbol{K}_{p x} \Delta \tilde{\boldsymbol{x}}$.

Considering (20) and differentiating (31) yields:

$$
\begin{aligned}
\dot{V}_{1} & =\Delta \ddot{\tilde{\boldsymbol{x}}}^{T} \Delta \dot{\tilde{\boldsymbol{x}}}+\Delta \dot{\tilde{\boldsymbol{x}}}^{T} \boldsymbol{K}_{p x} \Delta \tilde{\boldsymbol{x}} \\
& =-\left(\boldsymbol{K}_{v x} \Delta \dot{\tilde{\boldsymbol{x}}}+\boldsymbol{K}_{p x} \Delta \tilde{\boldsymbol{x}}\right) \Delta \dot{\tilde{\boldsymbol{x}}}+\Delta \dot{\tilde{\boldsymbol{x}}}^{T} \boldsymbol{K}_{p x} \Delta \tilde{\boldsymbol{x}} \\
& =-\Delta \dot{\tilde{\boldsymbol{x}}}^{T} \boldsymbol{K}_{v x} \Delta \dot{\tilde{\boldsymbol{x}}} .
\end{aligned}
$$

Define the second candidate Lyapunov function:

$V_{2}=V_{1}+\frac{1}{2} \Delta \dot{\boldsymbol{x}}^{T} \boldsymbol{M}_{e} \Delta \dot{\boldsymbol{x}}+\frac{1}{2} \Delta \boldsymbol{x}^{T} \boldsymbol{K}_{e} \Delta \boldsymbol{x}$.

The time derivative of (33) can be given as follows:

$$
\dot{V}_{2}=\dot{V}_{1}+\Delta \dot{\boldsymbol{x}}^{T} \boldsymbol{M}_{e} \Delta \ddot{\boldsymbol{x}}+\Delta \dot{\boldsymbol{x}}^{T} \boldsymbol{K}_{e} \Delta \dot{\boldsymbol{x}} .
$$

Combining (34) with (15) results in:

$$
\begin{aligned}
\dot{V}_{2} & =\dot{V}_{1}+\Delta \dot{\boldsymbol{x}}^{T}\left(\Delta \boldsymbol{F}_{I}-\boldsymbol{D}_{e} \Delta \dot{\boldsymbol{x}}-\boldsymbol{K}_{e} \Delta \boldsymbol{x}\right)+\Delta \dot{\boldsymbol{x}}^{T} \boldsymbol{K}_{e} \Delta \boldsymbol{x} \\
& =\dot{V}_{1}+\Delta \dot{\boldsymbol{x}}^{T}\left(\Delta \boldsymbol{F}_{I}-\boldsymbol{D}_{e} \Delta \dot{\boldsymbol{x}}\right) .
\end{aligned}
$$

From [37], we can know that:

$\Delta \dot{\boldsymbol{x}}=\boldsymbol{J}_{0} \Delta \dot{\boldsymbol{y}}$.

Since $\Delta \boldsymbol{F}_{I}$ lying in the null space of $\boldsymbol{J}_{0}^{T}$, it follows that:

$\boldsymbol{J}_{o}^{T} \Delta \boldsymbol{F}_{I}=0$.

Incorporating (36) and (37) into (35) yields:

$$
\begin{aligned}
\dot{V}_{2} & =\dot{V}_{1}+\Delta \dot{\boldsymbol{y}}^{T} \boldsymbol{J}_{o}^{T} \Delta \boldsymbol{F}_{I}-\Delta \dot{\boldsymbol{x}}^{T} \boldsymbol{D}_{e} \Delta \dot{\boldsymbol{x}} \\
& =\dot{V}_{1}-\Delta \dot{\boldsymbol{x}}^{T} \boldsymbol{D}_{e} \Delta \dot{\boldsymbol{x}} .
\end{aligned}
$$

Combining (38) with (32) results in:

$\dot{V}_{2}=-\Delta \dot{\tilde{\boldsymbol{x}}}^{T} \boldsymbol{K}_{v x} \Delta \dot{\tilde{\boldsymbol{x}}}-\Delta \dot{\boldsymbol{x}}^{T} \boldsymbol{D}_{e} \Delta \dot{\boldsymbol{x}}$

As $\boldsymbol{K}_{v x}$ and $\boldsymbol{D}_{e}$ are specified as symmetric positive-definite matrixes, $\dot{V}_{2}$ is negative semi-definite. With Lyapunov's second method and LaSalle invariance principle, the asymptotic stability of Eqs. (15) and (20) is ensured, namely, $\dot{V}_{2} \leq 0, \boldsymbol{F}_{I} \rightarrow \boldsymbol{F}_{I d}, \boldsymbol{x}_{r} \rightarrow \boldsymbol{x}_{d}$ and $\boldsymbol{x}_{a} \rightarrow \boldsymbol{x}_{r}$ for $t \rightarrow \infty$.

Define the third candidate Lyapunov function:

$V_{3}=V_{2}+\frac{1}{2} \boldsymbol{s}^{T} \boldsymbol{M}_{O I} \boldsymbol{s}$

Differentiating (40) yields:

$\dot{V}_{3}=\dot{V}_{2}+\boldsymbol{s}^{T} \boldsymbol{M}_{o I} \dot{\boldsymbol{s}}+\frac{1}{2} \boldsymbol{s}^{T} \dot{\boldsymbol{M}}_{o I} \boldsymbol{s}$.

Consider $\dot{\boldsymbol{M}}_{\boldsymbol{o}}=2 \boldsymbol{V}_{o}+\boldsymbol{S}$ where $\boldsymbol{S}$ is a skew-symmetric matrix, then combining the above equation with (27), (29) and (14), one has:

$$
\begin{aligned}
\dot{V}_{3}= & \dot{V}_{2}+\boldsymbol{s}^{T} \boldsymbol{M}_{O I} \dot{\boldsymbol{s}}+\frac{1}{2} \boldsymbol{s}^{T} \dot{\boldsymbol{M}}_{O I} \boldsymbol{s} \\
= & \dot{V}_{2}+\boldsymbol{s}^{T}\left(\boldsymbol{M}_{O I} \Delta \ddot{\boldsymbol{y}}+\boldsymbol{M}_{O I} \Lambda \Delta \dot{\boldsymbol{y}}\right)+\boldsymbol{s}^{T} \boldsymbol{V}_{o} \boldsymbol{s} \\
= & \dot{V}_{2}+\boldsymbol{s}^{T} \tilde{\boldsymbol{K}}_{O I} \Delta \boldsymbol{y}+\boldsymbol{s}^{T} \tilde{\boldsymbol{D}}_{o I} \Delta \dot{\boldsymbol{y}}+\boldsymbol{s}^{T} \boldsymbol{F}_{\text {env }} \\
& -\boldsymbol{s}^{T} \hat{\boldsymbol{K}}_{O I} \Delta \boldsymbol{y}-\boldsymbol{s}^{T} \hat{\boldsymbol{D}}_{o I} \Delta \dot{\boldsymbol{y}}+\boldsymbol{s}^{T} \boldsymbol{M}_{o} \boldsymbol{\Lambda} \Delta \dot{\boldsymbol{y}}+\boldsymbol{s}^{T} \boldsymbol{V}_{o} \boldsymbol{s} \\
\leq & \dot{V}_{2}+\boldsymbol{s}^{T} \tilde{\boldsymbol{K}}_{O I} \Delta \boldsymbol{y}+\boldsymbol{s}^{T} \tilde{\boldsymbol{D}}_{o I} \Delta \dot{\boldsymbol{y}}+\boldsymbol{s}^{T} \boldsymbol{M}_{o} \boldsymbol{\Lambda} \Delta \dot{\boldsymbol{y}}+\boldsymbol{s}^{T} \boldsymbol{V}_{o} \boldsymbol{s} .
\end{aligned}
$$

Out of consistency of the above expressions and the following equations, discrete form of $V_{3}$ is given as:

$$
\begin{aligned}
\Delta V_{3} \leq & \Delta V_{2}+\int_{t-T}^{t}\left(\boldsymbol{s}^{T} \tilde{\boldsymbol{K}}_{o I} \Delta \boldsymbol{y}+\boldsymbol{s}^{T} \tilde{\boldsymbol{D}}_{o I} \Delta \dot{\boldsymbol{y}}\right. \\
& \left.+\boldsymbol{s}^{T} \boldsymbol{M}_{0} \boldsymbol{\Lambda} \Delta \dot{\boldsymbol{y}}+\boldsymbol{s}^{T} \boldsymbol{V}_{o} \boldsymbol{s}\right) d \tau .
\end{aligned}
$$

To guarantee the asymptotical stability of the whole control system and minimize the energy cost of biomimetic adaption procedure, the overall Lyapunov function can be described by:

$V=V_{3}+V_{a}$

$V_{a}=V_{a k}+V_{a d}$

$V_{a k}=\frac{1}{2} \int_{t-T}^{t} \operatorname{vec}\left(\tilde{\boldsymbol{K}}_{o I}(\tau)\right)^{T} \cdot\left(\boldsymbol{I} \otimes \boldsymbol{Q}_{K}\right)^{-1} \cdot \operatorname{vec}\left(\tilde{\boldsymbol{K}}_{o I}(\tau)\right) d \tau$

$V_{a d}=\frac{1}{2} \int_{t-T}^{t} \operatorname{vec}\left(\tilde{\boldsymbol{D}}_{o I}(\tau)\right)^{T} \cdot\left(\boldsymbol{I} \otimes \boldsymbol{Q}_{D}\right)^{-1} \cdot \operatorname{vec}\left(\tilde{\boldsymbol{D}}_{o I}(\tau)\right) d \tau$

where $\otimes$ denotes Kronecker product, $\operatorname{vec}(\boldsymbol{M})$ is the column vectorization operator that transforms the matrix $\boldsymbol{M}$ into column vector. This definition actually describes the tendency to stability and the ultimate convergence of the impedance parameter. 


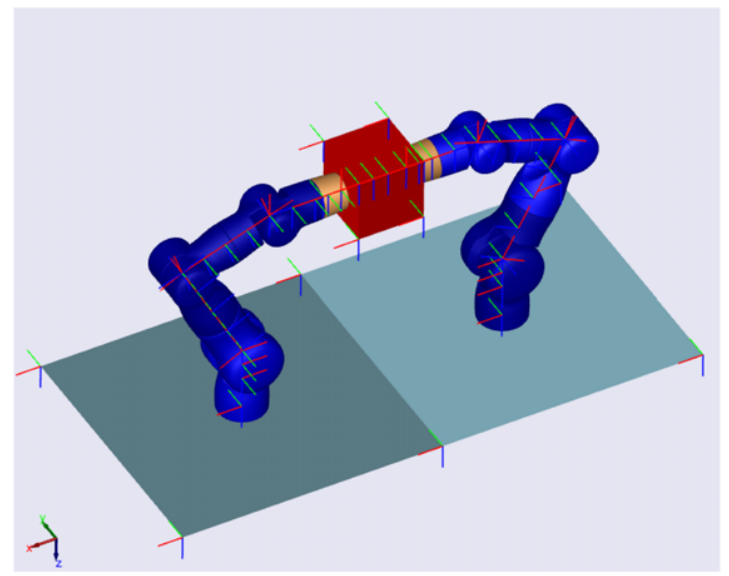

Fig. 3. Virtual model of the dual-arm system.

Then the difference of $V$ can be given as follows:

$$
\begin{aligned}
\Delta V=\Delta & V_{3}+\Delta V_{a k}+\Delta V_{a d} \\
\Delta V_{a k}= & \frac{1}{2} \int_{t-T}^{t}\left\{\left[\operatorname{vec}\left(\tilde{\boldsymbol{K}}_{o I}(\tau)\right)^{T} \cdot\left(\boldsymbol{I} \otimes \boldsymbol{Q}_{K}\right)^{-1} \cdot \operatorname{vec}\left(\tilde{\boldsymbol{K}}_{o I}(\tau)\right)\right.\right. \\
& -\operatorname{vec}\left(\tilde{\boldsymbol{K}}_{o I}(\tau-T)\right)^{T} \cdot\left(\boldsymbol{I} \otimes \boldsymbol{Q}_{K}\right)^{-1} \\
& \left.\left.\cdot \operatorname{vec}\left(\tilde{\boldsymbol{K}}_{o I}(\tau-T)\right)\right]\right\} d \tau \\
\Delta V_{a d}= & \frac{1}{2} \int_{t-T}^{t}\left\{\left[\operatorname{vec}\left(\tilde{\boldsymbol{D}}_{o I}(\tau)\right)^{T} \cdot\left(\boldsymbol{I} \otimes \boldsymbol{Q}_{D}\right)^{-1} \cdot \operatorname{vec}\left(\tilde{\boldsymbol{D}}_{o I}(\tau)\right)\right.\right. \\
& -\operatorname{vec}\left(\tilde{\boldsymbol{D}}_{o I}(\tau-T)\right)^{T} \cdot\left(\boldsymbol{I} \otimes \boldsymbol{Q}_{D}\right)^{-1} \\
& \left.\left.\cdot \operatorname{vec}\left(\tilde{\boldsymbol{D}}_{o I}(\tau-T)\right)\right]\right\} d \tau .
\end{aligned}
$$

When the gain parameters are chosen to satisfy the formula

$$
\gamma \operatorname{tr}\left(\boldsymbol{K}_{o I}^{T} \tilde{\boldsymbol{K}}_{o I}\right)+\gamma \operatorname{tr}\left(\boldsymbol{D}_{o I}^{T} \tilde{\boldsymbol{D}}_{o I}\right)+\boldsymbol{s}^{T} \boldsymbol{M}_{0} \boldsymbol{\Lambda} \Delta \dot{\boldsymbol{y}}+\boldsymbol{s}^{T} \boldsymbol{V}_{o} \boldsymbol{s} \leq 0 .
$$

Then the asymptotic stability of this adaptive controller and the convergence of the parameters are ensured. Detailed derivation is given in Appendix A.

\section{Simulations}

Here we use the toolbox SimMechanics in Simulink with ode45 solver to implement the modeling of the physical system with the proposed controller. This virtual model (as shown in Fig. 3) consists of three parts: the dual-arm cooperative 7-DOF humanoid manipulators, the dynamics of an object with specified parameters and the contact-force model between the two end-effectors and the object.

The model parameters of these two manipulators, which are listed in Table 1, are given to coincide with the physical system named Robonaut in HIT. Two manipulators share the same set of parameters.

The object manipulated by this virtual dual-arm system is a cube whose parameters are $1 \mathrm{~kg}$, diag $[0.4,0.4,0.4] \mathrm{kg} \mathrm{m}^{2}, 0.1 \mathrm{~m}$ for mass, inertia matrix and edge length respectively.

To depict the contact forces between the two end-effectors (disks) and the object in virtual simulation, penalty function method is adopted as the mathematic model. This approach is one

\begin{tabular}{|c|c|c|}
\hline Identifier & Length (m) & Mass/Inertia matrix \\
\hline 1 & 0.1 & $\begin{array}{l}3.47 \mathrm{~kg} \\
\operatorname{diag}[0.012,0.010,0.009] \mathrm{kg} \mathrm{m}^{2}\end{array}$ \\
\hline 2 & 0.2 & $\begin{array}{l}2.37 \mathrm{~kg} \\
\operatorname{diag}[0.020,0.025,0.023] \mathrm{kg} \mathrm{m}^{2}\end{array}$ \\
\hline 3 & 0.2 & $\begin{array}{l}2.25 \mathrm{~kg} \\
\operatorname{diag}[0.03,0.008,0.009] \mathrm{kg} \mathrm{m}^{2}\end{array}$ \\
\hline 4 & 0.14 & $\begin{array}{l}1.79 \mathrm{~kg} \\
\operatorname{diag}[0.002,0.044,0.044] \mathrm{kg} \mathrm{m}^{2}\end{array}$ \\
\hline 5 & 0.14 & $\begin{array}{l}1.72 \mathrm{~kg} \\
\operatorname{diag}[0.003,0.003,0.003] \mathrm{kg} \mathrm{m}^{2}\end{array}$ \\
\hline 6 & 0.14 & $\begin{array}{l}1.80 \mathrm{~kg} \\
\operatorname{diag}[0.002,0.005,0.005] \mathrm{kg} \mathrm{m}^{2}\end{array}$ \\
\hline 7 & 0.1 & $\begin{array}{l}1.0 \mathrm{~kg} \\
\operatorname{diag}[0.003,0.002,0.002] \mathrm{kg} \mathrm{m}^{2}\end{array}$ \\
\hline
\end{tabular}
of the most commonly used algorithms to calculate and model the contact force. It is assumed that the contact plane which is perpendicular to the axial direction of the end-effector can penetrate the object. The contact force is zero when the object is not penetrated, while non-zero contact force acting at the
Table 1

Model parameters of the two manipulators.

two arms and object are generated when it is done. The penalty function is described as:

$f(x)=k f_{1}(x)+d f_{2}(x, \dot{x})$

$f_{1}(x)=\max (0, x)$

$f_{2}(x, \dot{x})= \begin{cases}0 & x \leq 0 \\ \dot{x} & x>0\end{cases}$

where $k$ and $d$ are positive constants that represent the stiffness and damping of the contact surface respectively. $f$ denotes the contact force acted on the object exerted by the manipulators. $x$ expresses the relative distance between the ends of the manipulators and the object, $x$ is positive when the contact surface is penetrated and vice versa.

The stiffness and damping of this linear spring model are chosen as $10000 \mathrm{~N} / \mathrm{m}$ and $25 \mathrm{~kg} \mathrm{~s}^{-1}$.

This object has 6 DOFs. To simplify the whole simulation, we set position constrains in all DOFs but one horizontal direction which coincides with that of the contact force.

To verify the effectiveness of the proposed method, simulations with fixed and adaptive impedance parameters are implemented respectively. For the general object impedance scheme, the set of fixed parameters are chosen as follows:

$K=1, \quad K_{o I}=50 \mathrm{~N} / \mathrm{m}, \quad D_{o I}=5 \mathrm{~kg} / \mathrm{s}, \quad M_{e}=0.2 \mathrm{~kg}$,

$F_{I d}=20 \mathrm{~N}, \quad D_{e}=500 \mathrm{~kg} \mathrm{~s}^{-1}, \quad K_{e}=200 \mathrm{~N} / \mathrm{m}$.

For the biomimetic object impedance controller proposed here, the parameters are set by:

$K_{o I}^{*}=10 \mathrm{~N} / \mathrm{m}, \quad D_{o I}^{*}=8 \mathrm{~kg} / \mathrm{s}, \quad \Lambda=5$

$Q_{k}=60 \times 10^{3}, \quad Q_{D}=3 \times 10^{3}, \quad \gamma=0.5 \times 10^{-3}$.

It should be noted that $K_{o I}^{*}$ and $D_{o I}^{*}$ are initial values of the stiffness and damping of the object impedance, they are both critical to the initial stability when this novel control scheme is applied to simulation or physical experiment.

Both of the two schemes share the same parameters of the internal impedance and the inner controller for the dual-arm system. The parameters of Cartesian closed-loop control and jointspace closed control are given as:
$\boldsymbol{K}_{p x}=20 \boldsymbol{I}_{6}, \quad \boldsymbol{K}_{v x}=5 \boldsymbol{I}_{6}$
$\boldsymbol{K}_{p q}=\operatorname{diag}[200,150,150,150,80,100,50]$
$\boldsymbol{K}_{v q}=\operatorname{diag}[30,25,25,10,15,15,10]$.

Then an environmental force is commanded, as is shown in Fig. 4. Step forces of $5 \mathrm{~N}$ and $10 \mathrm{~N}$ (static force simulation) are applied on the object's center of mass of the object at $0 \mathrm{~s}$ and $10 \mathrm{~s}$ respectively, and then last for $5 \mathrm{~s}$. Another sine-wave force (high-frequency force simulation) is applied between $20 \mathrm{~s}$ and 

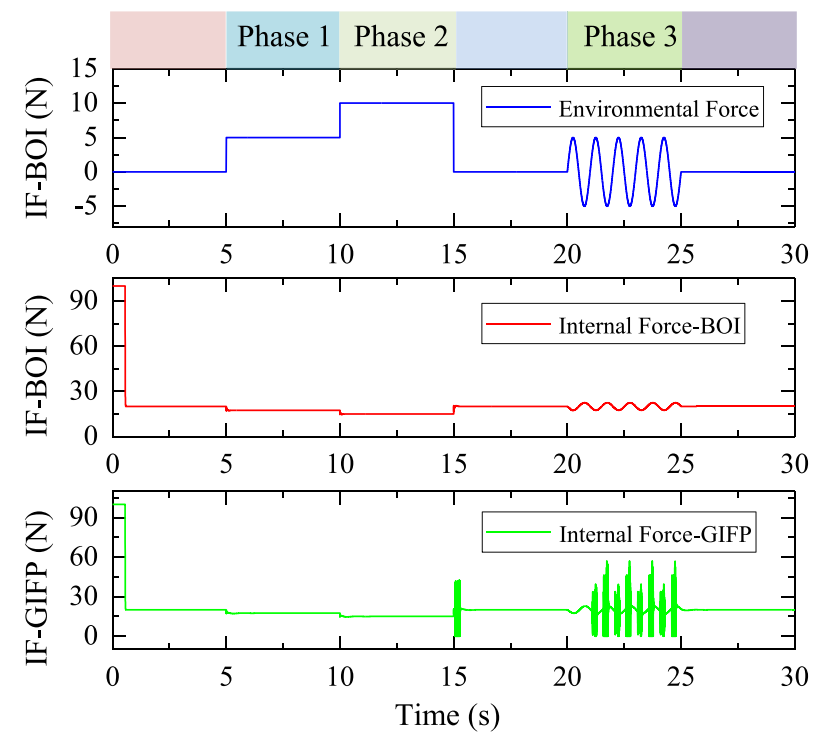

(a) Environmental force and internal forces exerted by two schemes.

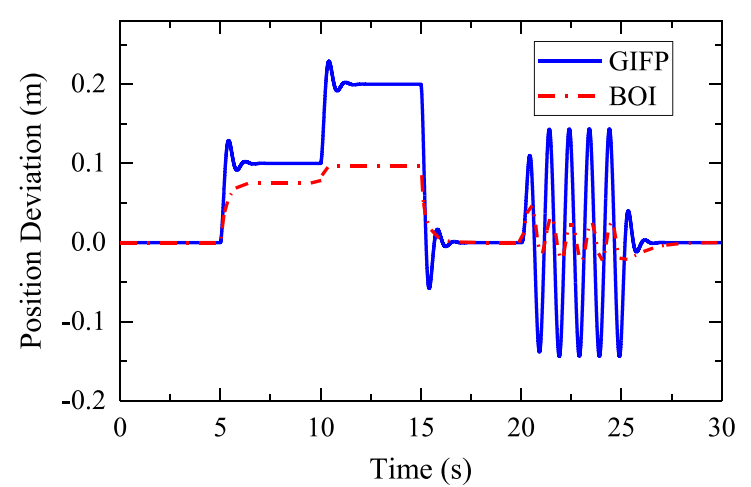

(b) Object position deviation with BOI and GIFP.

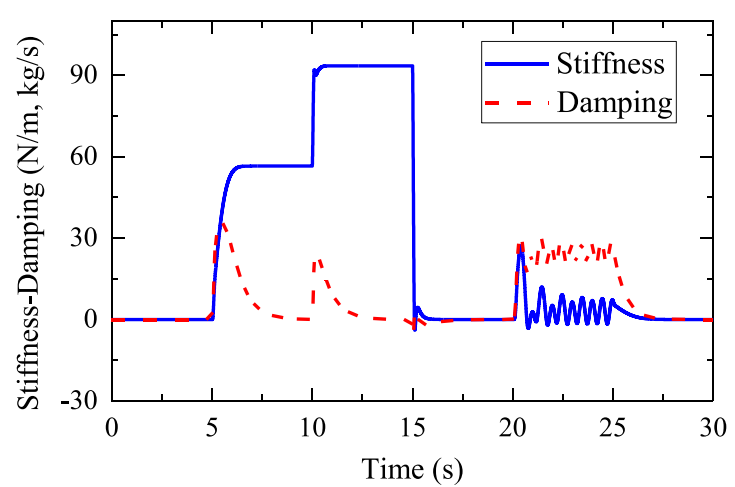

(c) Stiffness and damping adaption with BOI scheme.

Fig. 4. Simulation results of Biomimetic object impedance (BOI) and General impedance with fixed parameters (GIFP) schemes.

25 s. Internal forces exerted by manipulators are also exhibited in this figure when BOI (biomimetic object impedance scheme) and GIFP (general impedance scheme with fixed parameters) are adopted respectively. Compared with GIFP, BOI can ensure a much smoother variation of the internal force tracking when external disturbance changes.

The object deviations with these two schemes are also presented in Fig. 4. It should be mentioned that the object impedance parameters of the GIFP are tuned and chosen to achieve relatively the same performance compared to BOI in phase 1. GIFP may be able to obtain similar performance like BOI (in phase 1) but would generally underperform the BOI (in phases 2 and 3 ) when the external disturbance changes due to the lack of adaption and flexibility. Simulation results show superiority of BOI scheme. The magnitude of the deviation of the object's position is much more reduced. Moreover, due to the adaption of the parameters of the object impedance, the linear relationship between displacement and static force is no longer applicable.

The adaption of stiffness and damping of the BOI are exhibited in subpanel c of Fig. 4. When the disturbance is relatively stable (phase 1 and phase 2), the adaption of stiffness plays a major role, different magnitude of static force would build different stiffness. When the disturbance changes dramatically (phase 3 ), the adaption of damping predominates. This property confirms the expectation of the proposed BOI in this paper.
To further study the influence brought in by the parameters on the object impedance, three different sets of parameters are chosen as: (a) $Q_{k}=60 \times 10^{3}, Q_{D}=3 \times 10^{3}, \gamma=0.5 \times 10^{-3}$; (b) $Q_{k}=120 \times 10^{3}, Q_{D}=6 \times 10^{3}, \gamma=0.5 \times 10^{-3}$; (c) $Q_{k}=60 \times 10^{3}, Q_{D}=3 \times 10^{3}, \gamma=2 \times 10^{-3}$.

Simulation results are presented in Fig. 5, from which the following conclusions can be drawn: $Q_{k}$ and $Q_{D}$ influence the convergence rate of object stiffness and damping respectively, parameter $\gamma$ not only affects the steady-state value of stiffness and damping but also influences their convergence rate.

Throughout the simulation, the effectiveness of the novel biomimetic controller is demonstrated.

\section{Implementation and experiments}

The proposed algorithm was tested on a dual-arm robot named HIT-Robonaut, which is equipped with two 7-DOF humanoid manipulators (as shown in Fig. 6). The hardware architecture of this dual-arm cooperative system is shown in Fig. 7. A series of experiments are carried out to demonstrate the advantages of the adaptive object impedance strategy over other conventional object impedance scheme, such as the characteristics dealing with unstable interactions. The two manipulators are both mounted on the fixed base and grasping a box whose size is $0.40 \mathrm{~m} \times 0.25 \mathrm{~m} \times$ $0.25 \mathrm{~m}$. A brief description of this dual-arm system is given in Appendix B. 


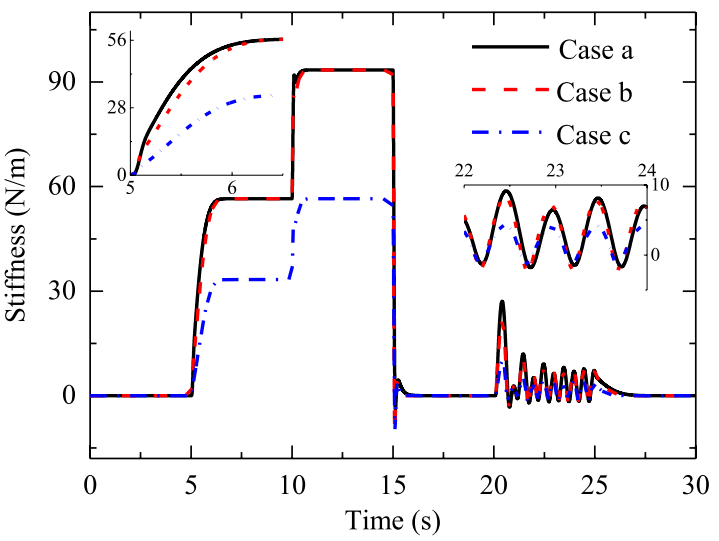

(a) Adaption of stiffness.

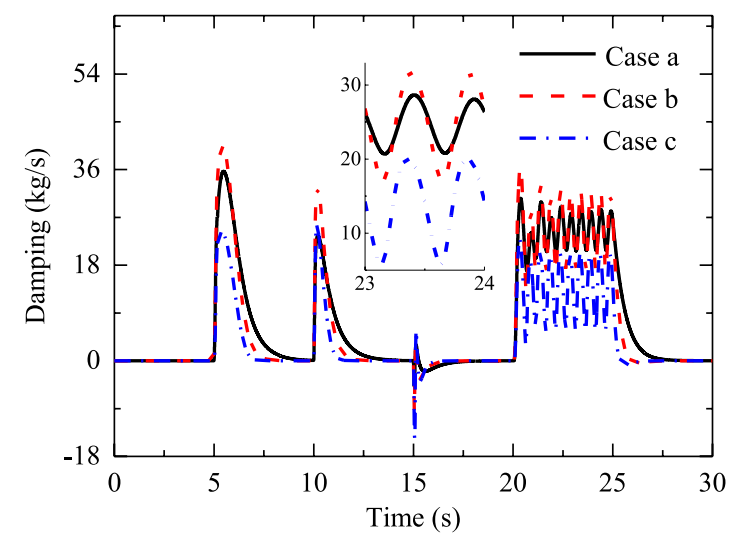

(b) Adaption of damping.

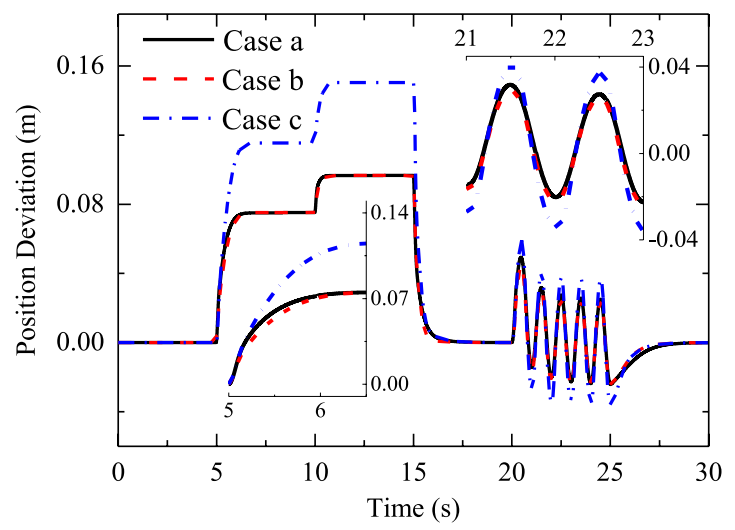

(c) Object position deviation.

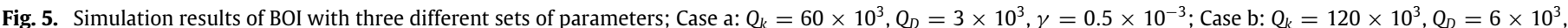
$\gamma=0.5 \times 10^{-3}$; Case c: $Q_{k}=60 \times 10^{3}, Q_{D}=3 \times 10^{3}, \gamma=2 \times 10^{-3}$.

It is worth mentioning that to reduce the amount of the quantities indirectly measured and increase the credibility of the experimental results, here we use the API Laser Tracker III to observe and record the pose and motion of the object. The static/dynamic absolute accuracy are $\pm 5 \mathrm{ppm}$ and $\pm 10 \mathrm{ppm}$, respectively.

Since the proposed algorithm enables a consistency in all directions, then to simplify the procedure, the motion characteristics of the object along one certain direction is mainly studied for each following experiment.

\subsection{Static interaction experiments}

To obtain the static response characteristic, we apply artificial static interference on the object held by the dual-arm system. First of all, two 7-DOF humanoid manipulators grasp the box tightly and keep it still. Then two weights are gently stacked on the object respectively, this process is equivalent to applying a static interference to the box and minimizing the dynamic disturbance term simultaneously. The process of stacking weighs is carried out with two parts. At the first step, we put the weight with the mass of $0.5 \mathrm{~kg}$ on the box, then stack another weight above the former one. To highlight the trend that the object impedance characteristic demonstrates along one typical axis, the two weights should be put at the object's center of mass as accurately as possible, this can ensure the minimization of the introduced disturbance acting on the box along other axes. We do not need to mix them up because the consistence of the impedance characteristics along the three axes is guaranteed by Eq. (12).

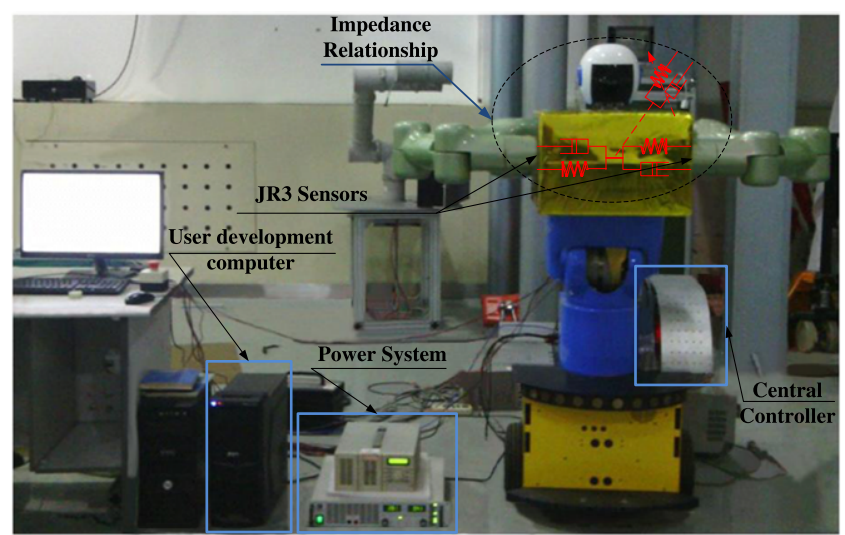

Fig. 6. Experimental setup for HIT-Robonaut grasping a box.

To intuitively show the relationship between the environmental disturbance and the adaptive stiffness \& damping, the external forces should be displayed. Since the applied force exerted by the end-effector of each manipulator can be obtained by real-time measuring of the two JR3s, the external forces (the object's dynamics is properly ignored because it is much smaller than the external forces) exhibited in the following figures can be obtained by mapping transformations and some proper computations.

According to Eqs. (3) and (4), also considering the aforementioned size parameters and the centroid position of the box, the 


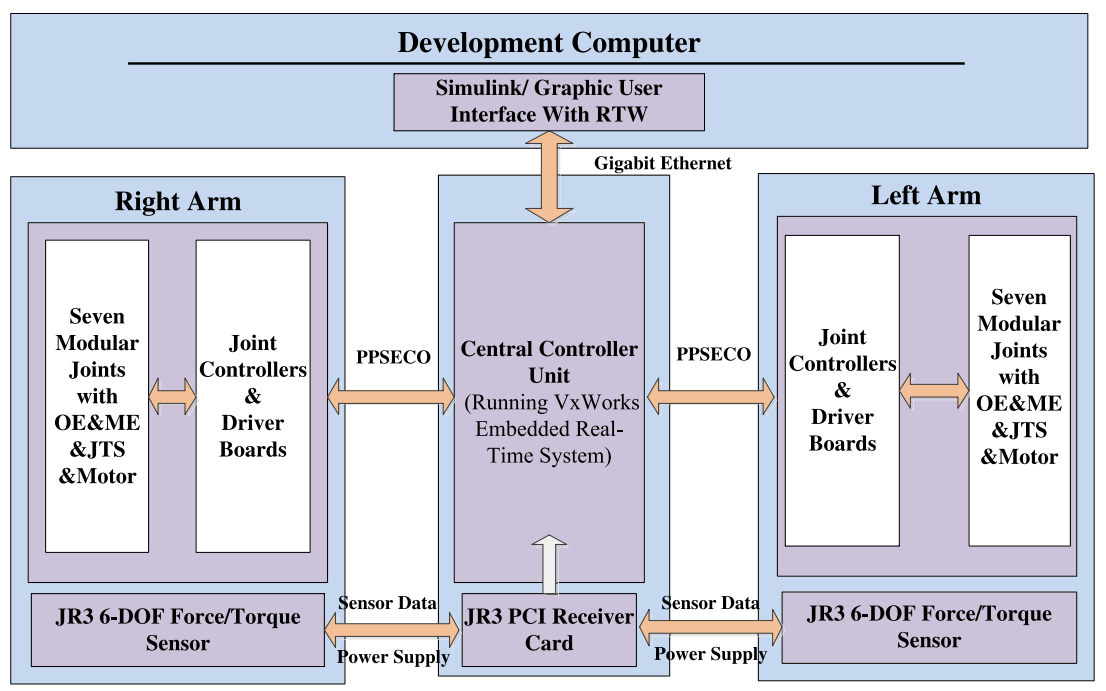

Fig. 7. Hardware Architecture of the dual-arm cooperative system.

grasp matrix here is then given by the following equation:

$\boldsymbol{J}_{0}^{T}=\left[\begin{array}{cccccccc} & \boldsymbol{I}_{\mathbf{3}} & & \mathbf{0}_{\mathbf{3}} & & \boldsymbol{I}_{\mathbf{3}} & & \mathbf{0}_{\mathbf{3}} \\ 0 & 0 & 0 & & 0 & 0 & 0 & \\ 0 & 0 & -0.2 & \boldsymbol{I}_{\mathbf{3}} & 0 & 0 & 0.2 & \boldsymbol{I}_{\mathbf{3}} \\ 0 & 0.2 & 0 & & 0 & -0.2 & 0 & \end{array}\right]$.

First of all, general object impedance strategy with fixed parameters is adopted. The parameters of the object impedance are set to: $\boldsymbol{K}_{\boldsymbol{o I}}=100 \boldsymbol{I}_{\mathbf{6}} \mathrm{N} / \mathrm{m} ; \boldsymbol{D}_{\boldsymbol{o I}}=15 \boldsymbol{I}_{\mathbf{6}} \mathrm{kg} / \mathrm{s} ; \boldsymbol{F}_{\boldsymbol{I} \boldsymbol{d}}=20 \mathrm{~N}$. Experimental results are shown in Fig. 8. According to the forces/torques measured by the two JR3 sensors and through proper grasp mapping, we can know that the introduced weight at the first step brings the object a $(-0.5,4.8,0.6) \mathrm{N}$ static interference force and at the second step exerts a $(-1.4,9,1.0) \mathrm{N}$ equivalent force respectively. Obviously the non-y-axial components of the external force applying on the box are neither zero. This is due to the non-coincidence between the centers of the weights and the box. With the above static forces, the object's position derivations from its initial position are $(-5,48,4.1) \mathrm{mm}$ and $(-9.1,90.3,9.5) \mathrm{mm}$ (as shown in subpanel (c) of Fig. 8). It should be noted that the approximate linear relationship exists between the external static force and the deviation. After the removal of the weights, the box returns to its initial position at 0 .

Secondly, parameters adopted in the proposed adaptive object impedance are $\boldsymbol{Q}_{\boldsymbol{K}}=6 \times 10^{4} \mathbf{I}_{\mathbf{6}}, \boldsymbol{Q}_{\boldsymbol{D}}=3 \times 10^{3} \mathbf{I}_{\mathbf{6}}, \boldsymbol{\Lambda}=5 \mathbf{I}_{\mathbf{6}}, \gamma=$ $0.25 \times 10^{-3}, \boldsymbol{K}_{\boldsymbol{o I}}^{*}=30 \boldsymbol{I}_{\mathbf{6}} \mathrm{N} / \mathrm{m}, \boldsymbol{D}_{\boldsymbol{o I}}^{*}=10 \boldsymbol{I}_{\mathbf{6}} \mathrm{kg} / \mathrm{s}$. The experimental results are also given in Fig. 8. The two weights stacked on the box are equivalent to applying to the object a $(0.37,4.75,0.38) \mathrm{N}$ and a $(0.8,9.4,1.18) \mathrm{N}$ static interference force respectively (as illustrated in subpanel (b) of Fig. 8). With these two equivalent static interference forces, the position of the box is deviated to $(7,49,9) \mathrm{mm}$ and $(12,61,17) \mathrm{mm}$ from its initial position, the external force along the $y$-axis exhibits a nonlinear relationship with the corresponding displacement along the same axis. This phenomenon is caused by the accumulation of the stiffness of the object impedance which is tuned by the adaptive law. During the whole process, the stiffness increases to $(5,72,5) \mathrm{N} / \mathrm{m}$ and $(10,116,15) \mathrm{N} / \mathrm{m}$ respectively (as illustrated in subpanel (e) of Fig. 8). So the increment of displacement corresponding to the weights added on at the second step is smaller than the previous one. Meanwhile, from the results shown in subpanel (f) of Fig. 8, we can see that the damping of the object impedance varies only at the moment we drop the weights on the box and it returns to 0 when the system achieves stability. Also it is noted that the damping increases to a positive value at the instant of placing the weights on and decreases to a negative value when removing the weights out. How the damping varied depends on whether the current speed of the object benefits the return of the object to its initial position. Furthermore, internal force tracking results with these two schemes are shown in subpanel (g), which illustrates the fact that both of them have similar force tracking performance and can ensure bounded internal force when static disturbance exists.

To further verify the influence on the stiffness and damping of the object adaptive impedance brought by the parameter $\gamma$, two different values are assigned to it: (a) $\gamma=0.25 \times 10^{-3}$ and (b) $\gamma=0.50 \times 10^{-3}$. The experimental results are both shown in Fig. 9. Comparing the results of the two experiments, it is noted that the steady-state value of the stiffness along the direction of the interaction force at the time when the first weight is placed on are $69 \mathrm{~N} / \mathrm{m}$ and $39 \mathrm{~N} / \mathrm{m}$ respectively, the maximum damping are $25 \mathrm{~kg} / \mathrm{s}$ and $14 \mathrm{~kg} / \mathrm{s}$. After the second weight is stacked, the stable stiffness increases to $113 \mathrm{~N} / \mathrm{m}$ and $65 \mathrm{~N} / \mathrm{m}$, the maximum damping are $12 \mathrm{~kg} / \mathrm{s}$ and $7 \mathrm{~kg} / \mathrm{s}$. Actually, consistent with the simulation results in Section 4, the parameter $\gamma$ has a significant influence on the build-up speed of the stiffness and damping of the object impedance. Larger $\gamma$ may lead to a faster forgetting speed, thus establishing a smaller rate of convergence and stable value of the two key object impedance parameters.

In summary, with the presence of the static environmental interference, the adaptive object impedance control strategy mainly builds up the stiffness to suppress the displacement of the object when interacting with the static environment. Definitely, with the tunable parameters, we can get much faster rate of convergence and smaller steady-state deviation. This depends on the practical requirements.

\subsection{Dynamic interaction experiments}

Furthermore, this part will demonstrate the dynamic interaction experiments. Artificial dynamic interference is applied to the object to help obtain the dynamic response characteristic of the system with GIFP and BOI schemes. The two manipulators hold the box and keep it stationary beforehand, then we push and pull the clamped box along the $x$-axis several times to simulate the horizontal dynamic interference force. The variations of the object's position and internal forces with these two schemes are recorded and shown in subpanels (a)-(d) of Fig. 10. With the push-pull, the clamped box interacts with human along $x$-axis. The amplitude of this force is approximately $\pm 10 \mathrm{~N}$. As this interaction varies, the 


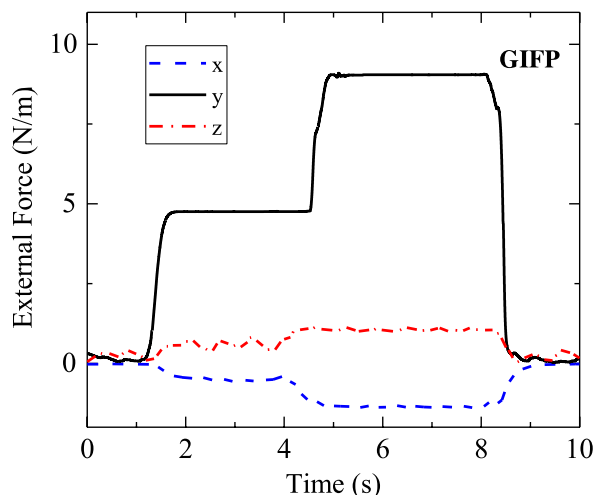

(a) External forces applied on the object with GIFP.

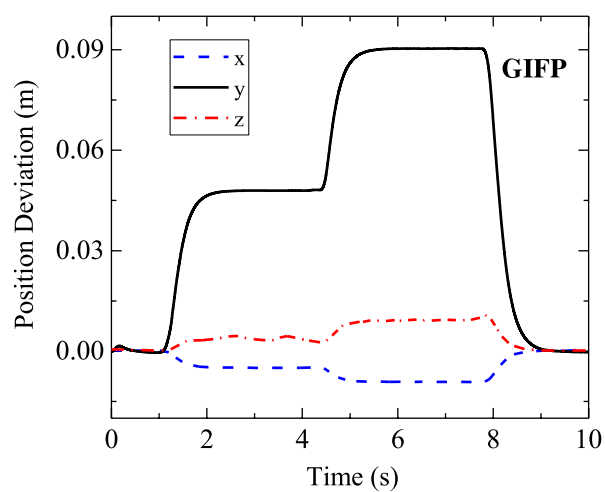

(c) Object position deviation with GIFP.

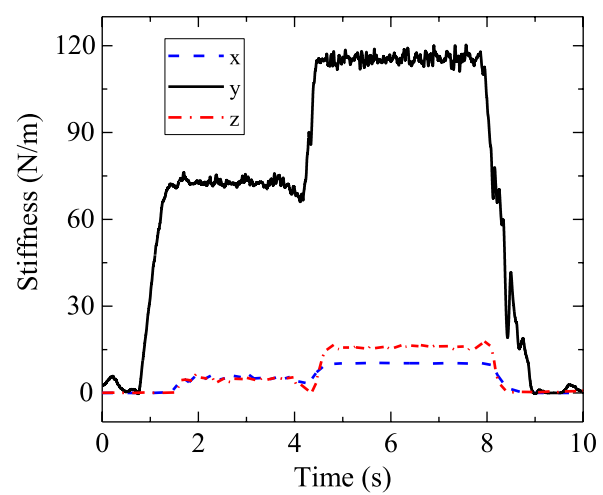

(e) Stiffness adaption with BOI scheme.

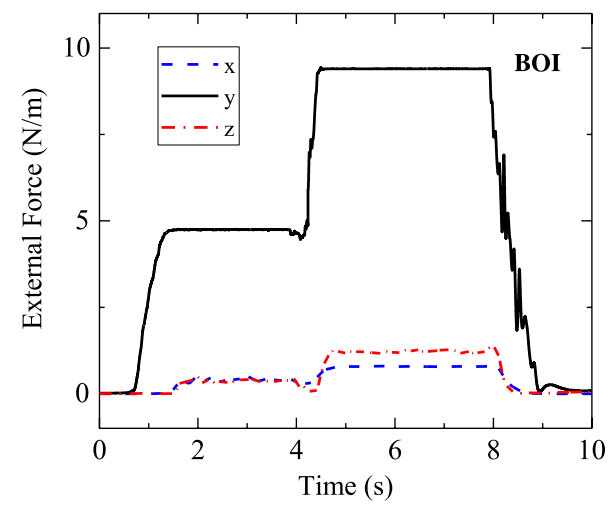

(b) External forces applied on the object with BOI.

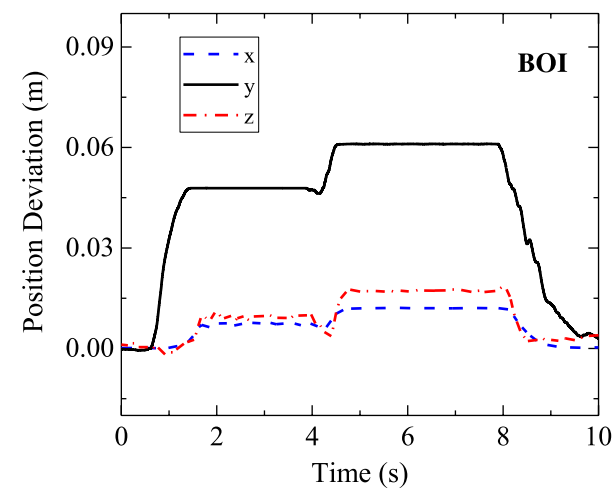

(d) Object position deviation with BOI.

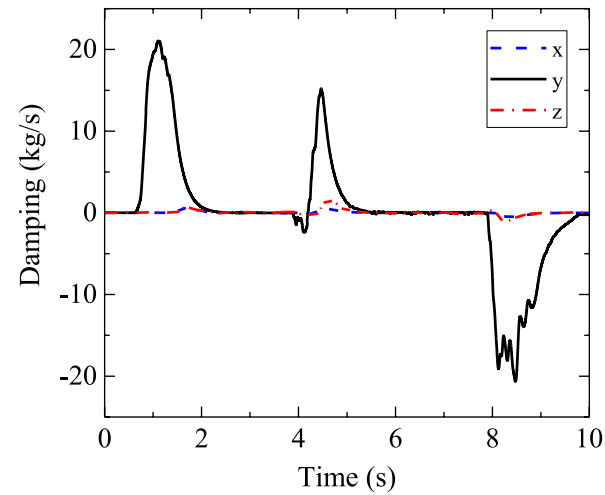

(f) Damping adaption with BOI scheme.

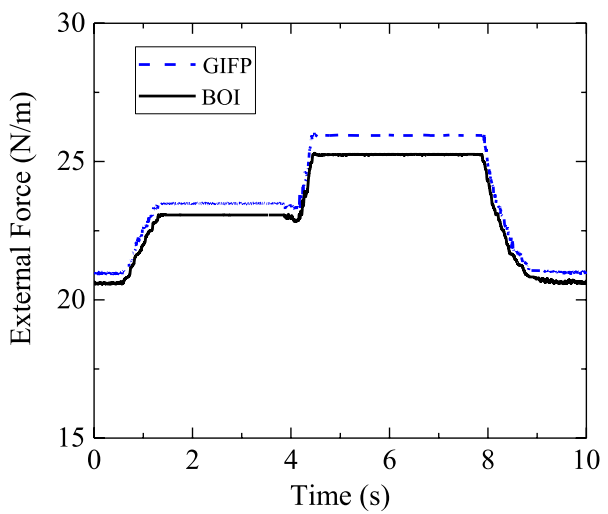

(g) Internal forces with GIFP and BOI.

Fig. 8. Experimental results of BOI and GIFP schemes with static interactions. 


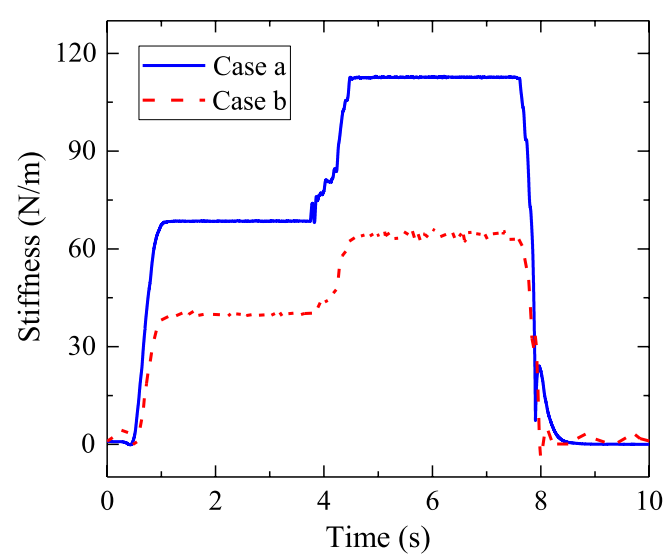

(a) Adaption of stiffness.

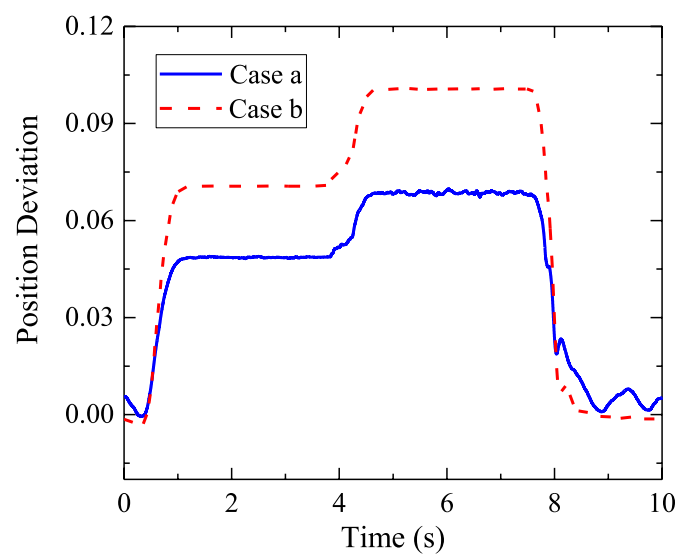

(c) Object position deviation.

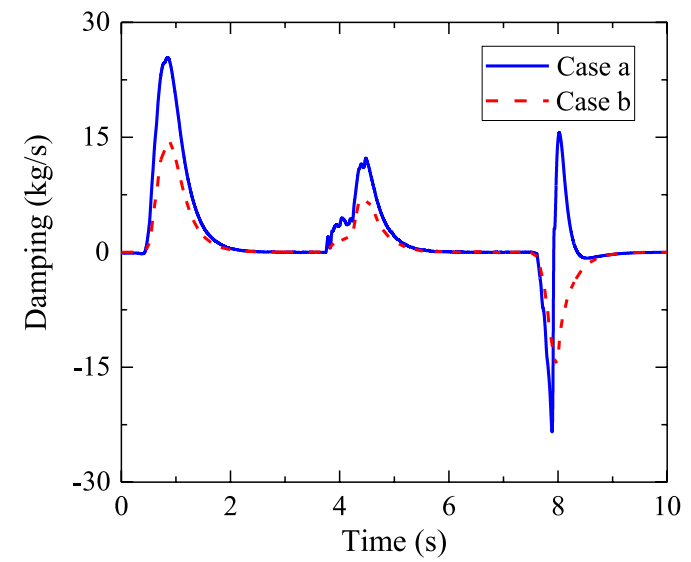

(b) Adaption of damping.

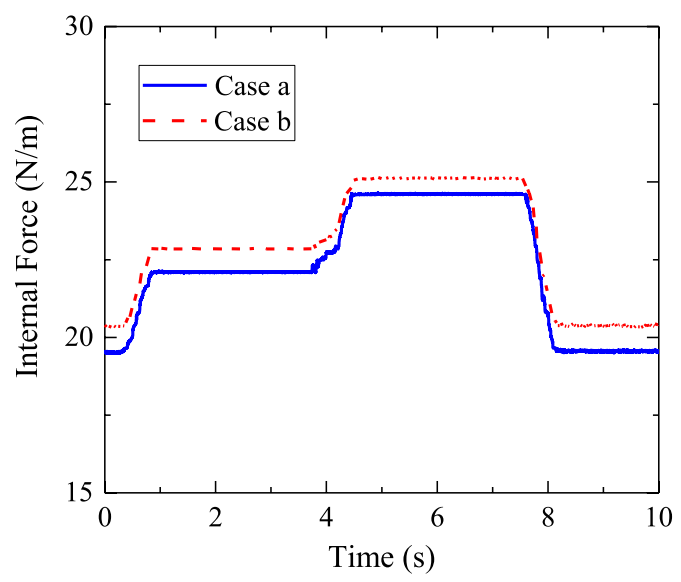

(d) Internal forces.

Fig. 9. Experimental results of BOI with two different sets of parameters; Case a: $\gamma=0.25 \times 10^{-3}$; Case b: $\gamma=0.5 \times 10^{-3}$.

box moves back and forth relative to the initial position in the range between -0.03 and $0.02 \mathrm{~m}$ when using BOI (as shown in subpanel (c) of Fig. 10), while the motion range with GIFP is from -0.1 to $0.1 \mathrm{~m}$. During the shaking, the stiffness of the BOI approximately increases to $10 \mathrm{~N} / \mathrm{m}$. This slight inconsistence between the results of simulation and that of this experiment is due to the asymmetry of the reciprocating force. In this case, the adaption of damping outstands. It increases to nearly $40 \mathrm{~kg} / \mathrm{s}$. This is because the adaptive object impedance strategy would build up the damping to suppress the variation of the object's speed. The internal force tracking results are shown in subpanel (b), from which we can know that the fluctuation of internal force with BOI is much smoother than that with GIFP when dynamic interaction exists.

\section{Discussion}

In this paper, a novel control scheme with biomimetic adaption of object impedance for dual-arm cooperative 7-DOF manipulators is proposed. The current study is based on the impedance architecture first proposed in [16]. We further introduce force tracking into the internal impedance level and biomimetic adaption mechanism into the object impedance level, which makes the scheme advantage over other related approaches in some aspects. Compared with [16], this controller exhibits explicit impedance behavior when the interaction with environment is weak, and shows accumulation property of the stiffness and damping accordingly. The adaption of stiffness plays a major role when the disturbance is relatively stable, whereas the adaption of damping predominates when disturbance changes dramatically. Furthermore, it can simultaneously minimize the energy cost of the adaptation process. Through tuning the adaptive parameters in the controller, we can readily achieve the desired performance. This human-like characteristic enables the object/dual-arm system to biomimetically deal with complex and unknown environmental disturbances. Also, it can avoid unnecessary energy cost to maintain high stiffness and damping when no interaction exists. Being different from [21], the adaptive mechanism used in our paper is to mainly achieve the adaption of the stiffness and damping of the object impedance rather than to generate new trajectories by the way of trials and learning rules, this helps the dual-arm system respond to the unknown interaction timely.

Although our method advances the object impedance control for dual-arm system, there still remain limitations and room for improvement as future works which can be focused on the following three aspects:

First, proper choice of the generalized inverse of the grasp matrix can totally decouple the external force and internal force and avoid inherent squeezing effect in motion-inducing force, but their corresponding object impedance and internal impedance cannot be dynamically decoupled since the response rate of a secondorder system is finite essentially. Whether dealing with the problem to the extent that the main goal can be achieved substantially from an engineering point of view or completely solving it theoretically by proposing an improved control architecture needs further discussion and experimental validation.

The second work direction is to transplant this algorithm to a more general case, the grasp of objects of different weights or with arbitrary flexibility.

When choosing the same grasp type, the only difference between heavy and light objects is the force magnitude (or 


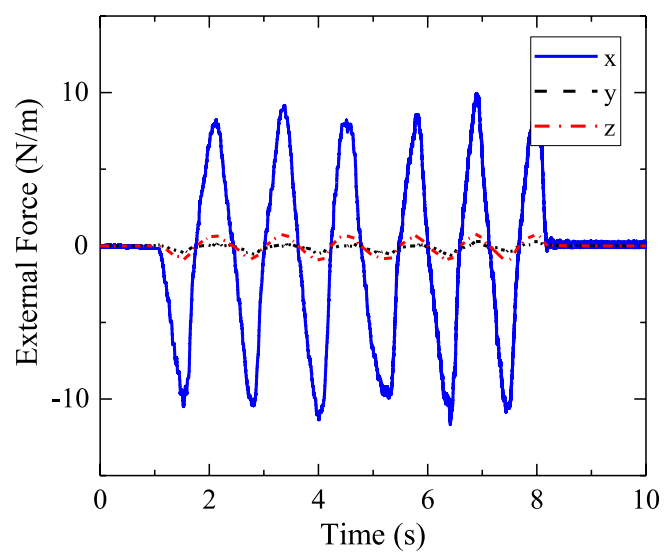

(a) External force applied on the object.

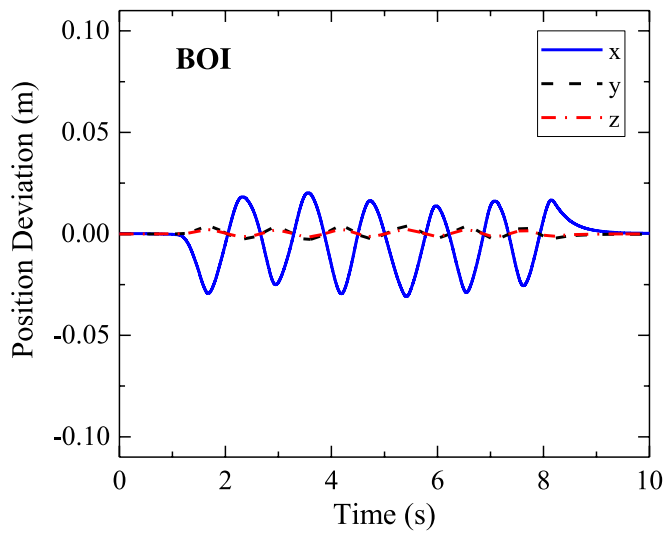

(c) Object position deviation with BOI.

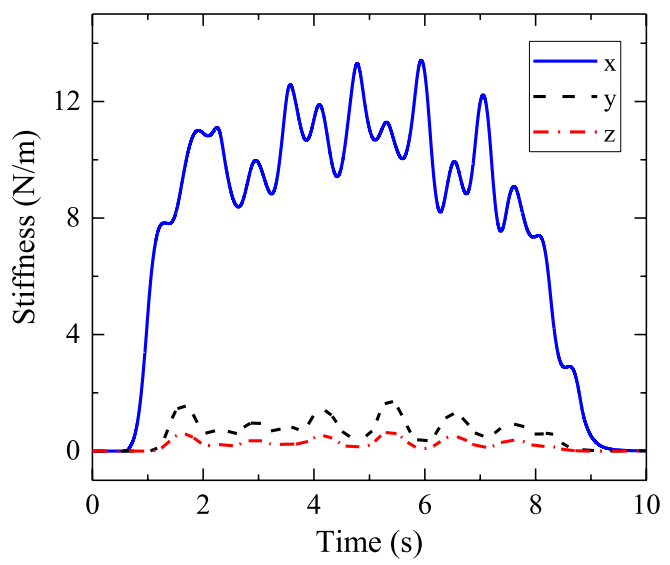

(e) Adaption of stiffness with BOI.

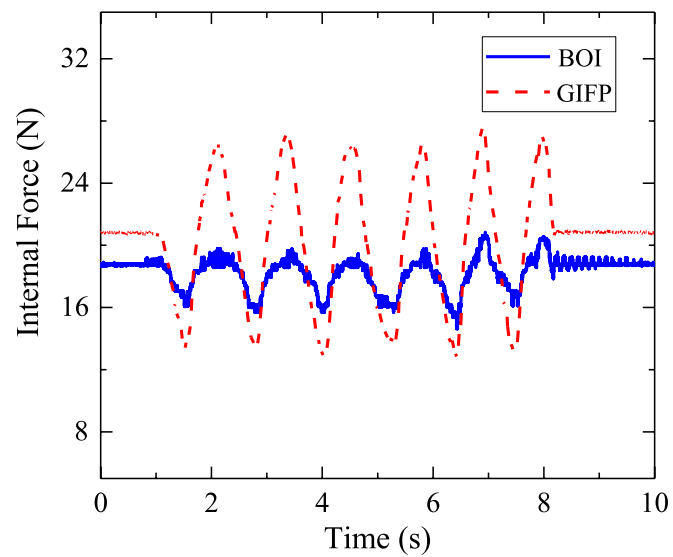

(b) Internal forces with BOI and GIFP.

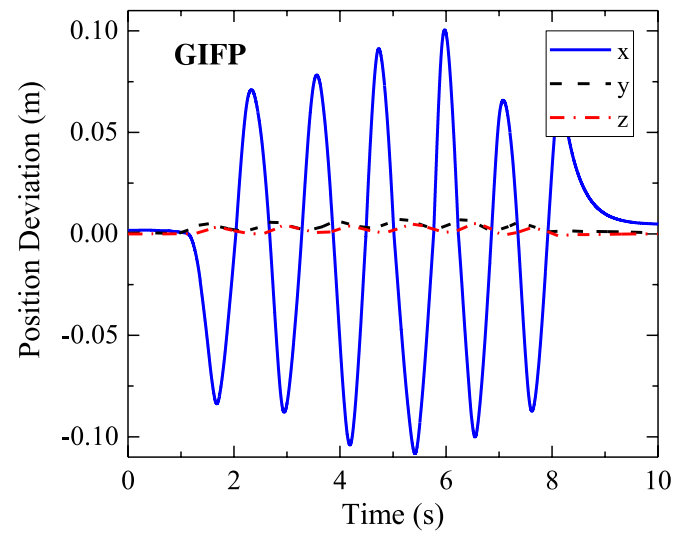

(d) Object position deviation with GIFP.

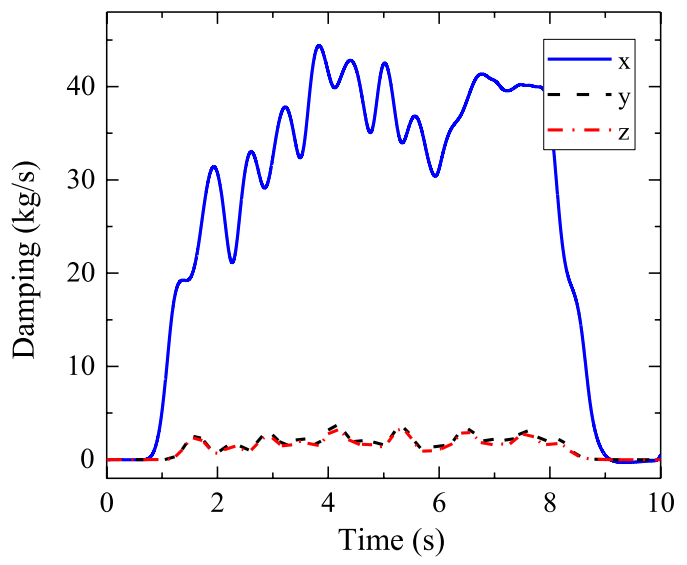

(f) Adaption of damping with BOI.

Fig. 10. Experimental results of BOI and GIFP schemes with dynamic interactions.

grasp stiffness) required if they are made of the same material, or put another way, share the same mechanical property. So the biomimetic object impedance control strategy can be easily transplanted to the grasp of objects of different weights under the premise of enough driving ability.

While this simple conclusion does not fit the case that soft or rigid object is grasped, the flexibility of the object would make the modeling more difficult. This problem should be divided into two cases according to the degree of flexibility and discussed respectively. The first case is that the stiffness of the object is relatively high. Then it is assumed that deformation of the object is very small, so the motion of the object can be approximately regarded as a rigid body. This assumption is reasonable when a rigid load is surrounded with bumpers or elastic materials. Another illustration of this assumption is multiple grippers grasping a rigid load, where the grippers possess compliance from installed flexible mechanisms [38]. The contact forces between the manipulators and the flexible object are modeled as gradients of nonlinear potentials which describe the deformations of the object. Namely, the kinematics of the object/dual-arm system needs no revision and only the modeling of the contact forces should be replaced compared with the analysis in our manuscript. The second case is a more general case in which the object has arbitrary flexibility. The flexible object can be decomposed into a rigid component, which represents the original shape, and a flexible component which represents the change in shape due to the deformation. This allows 
us to treat the motion of the object separately. Accordingly, the motion of the object comprises of rigid motion (its gross motion) and elastic motion. To simulate the flexible model numerically, the finite-element method can be used to discretize the flexible body as a regular discrete mesh of nodes. Then the deformation of all nodes can be defined separately [39]. In a word, both kinematics and dynamics need thorough reanalysis if we want to transplant the biomimetic controller to this case.

Finally, it is worth mentioning that friction between the endeffector and object brings no effect to the stable grasp according to the mechanical analysis. In physical experiment, however, it indeed contributes to the grasp success rate due to the nonideal grasp condition. This means that the internal impedance which determines the clamping force and further determines the friction does play a positive role. So the parameters of the internal impedance should be properly adjusted according to the object's weight. Further work is needed.

\section{Acknowledgments}

We would like to express our great appreciation to Dr. Yang Zhou for his sparkling idea and great help in the establishment of the platform. This work gets support from National Natural Science Foundation of China (NO. 51505098).

\section{Appendix A. Derivation}

Considering that the gain matrix $\boldsymbol{Q}_{\boldsymbol{K}}$ and $\boldsymbol{Q}_{\boldsymbol{d}}$ are symmetric, then with simple formula deformation, (48) and (49) can be rewritten as:

$$
\begin{aligned}
\Delta V_{a k}= & \frac{1}{2} \int_{t-T}^{t}\left\{\left[\operatorname{vec}\left(\tilde{\boldsymbol{K}}_{o I}(\tau)\right)^{T}-\operatorname{vec}\left(\tilde{\boldsymbol{K}}_{o I}(\tau-T)\right)^{T}\right]\right. \\
& \left.\cdot\left(\boldsymbol{I} \otimes \boldsymbol{Q}_{K}\right)^{-1} \cdot\left[\operatorname{vec}\left(\tilde{\boldsymbol{K}}_{o I}(\tau)\right)+\operatorname{vec}\left(\tilde{\boldsymbol{K}}_{o I}(\tau-T)\right)\right]\right\} d \tau \\
\Delta V_{a d}= & \frac{1}{2} \int_{t-T}^{t}\left\{\left[\operatorname{vec}\left(\tilde{\boldsymbol{D}}_{o I}(\tau)\right)^{T}-\operatorname{vec}\left(\tilde{\boldsymbol{D}}_{o I}(\tau-T)\right)^{T}\right]\right. \\
& \left.\cdot\left(\boldsymbol{I} \otimes \boldsymbol{Q}_{K}\right)^{-1} \cdot\left[\operatorname{vec}\left(\tilde{\boldsymbol{D}}_{o I}(\tau)\right)+\operatorname{vec}\left(\tilde{\boldsymbol{D}}_{o I}(\tau-T)\right)\right]\right\} d \tau .
\end{aligned}
$$

By combining (28) and (30), one has:

$$
\begin{aligned}
\Delta \tilde{\boldsymbol{K}}_{O I} & =-\Delta \boldsymbol{K}_{O I} \\
\Delta \tilde{\boldsymbol{D}}_{O I} & =-\Delta \boldsymbol{D}_{O I} .
\end{aligned}
$$

With simple formula deformation, we can get:

$$
\begin{aligned}
{\left[\operatorname{vec}\left(\tilde{\boldsymbol{K}}_{o I}(\tau)\right)^{T}-\operatorname{vec}\left(\tilde{\boldsymbol{K}}_{o I}(\tau-T)\right)^{T}\right] \cdot\left(\boldsymbol{I} \otimes \boldsymbol{Q}_{K}\right)^{-1} } \\
\\
\cdot\left[\operatorname{vec}\left(\tilde{\boldsymbol{K}}_{o I}(\tau)\right)+\operatorname{vec}\left(\tilde{\boldsymbol{K}}_{o I}(\tau-T)\right)\right] \\
=\operatorname{vec}\left(\tilde{\boldsymbol{K}}_{o I}(\tau)-\tilde{\boldsymbol{K}}_{o I}(\tau-T)\right)^{T} \cdot\left(\boldsymbol{I} \otimes \boldsymbol{Q}_{K}\right)^{-1} \\
\cdot \operatorname{vec}\left(2 \tilde{\boldsymbol{K}}_{o I}(\tau)-\tilde{\boldsymbol{K}}_{o I}(\tau)+\tilde{\boldsymbol{K}}_{o I}(\tau-T)\right) \\
=-\operatorname{vec}\left(\Delta \tilde{\boldsymbol{K}}_{o I}\right)^{T} \cdot\left(\boldsymbol{I} \otimes \boldsymbol{Q}_{K}\right)^{-1} \cdot \operatorname{vec}\left(\Delta \tilde{\boldsymbol{K}}_{o I}\right) \\
\quad+2 \operatorname{vec}\left(\Delta \tilde{\boldsymbol{K}}_{o I}\right)^{T} \cdot\left(\boldsymbol{I} \otimes \boldsymbol{Q}_{K}\right)^{-1} \cdot \operatorname{vec}\left(\tilde{\boldsymbol{K}}_{O I}(\tau)\right) .
\end{aligned}
$$

By folding (A.3) into (A.1) we can further simplify this equation

$$
\begin{aligned}
{[\operatorname{vec}} & \left.\left(\tilde{\boldsymbol{K}}_{o I}(\tau)\right)^{T}-\operatorname{vec}\left(\tilde{\boldsymbol{K}}_{o I}(\tau-T)\right)^{T}\right] \cdot\left(\boldsymbol{I} \otimes \boldsymbol{Q}_{K}\right)^{-1} \\
& \cdot\left[\operatorname{vec}\left(\tilde{\boldsymbol{K}}_{O I}(\tau)\right)+\operatorname{vec}\left(\tilde{\boldsymbol{K}}_{o I}(\tau-T)\right)\right] \\
= & -\operatorname{vec}\left(\Delta \tilde{\boldsymbol{K}}_{o I}\right)^{T} \cdot\left(\boldsymbol{I} \otimes \boldsymbol{Q}_{K}\right)^{-1} \cdot \operatorname{vec}\left(\Delta \tilde{\boldsymbol{K}}_{o I}\right) \\
& -2 \operatorname{vec}\left(\Delta \boldsymbol{K}_{o I}\right)^{T} \cdot\left(\boldsymbol{I} \otimes \boldsymbol{Q}_{K}\right)^{-1} \cdot \operatorname{vec}\left(\tilde{\boldsymbol{K}}_{o I}(\tau)\right) \\
= & -\operatorname{vec}\left(\Delta \tilde{\boldsymbol{K}}_{o I}\right)^{T} \cdot\left(\boldsymbol{I} \otimes \boldsymbol{Q}_{K}\right)^{-1} \cdot \operatorname{vec}\left(\Delta \tilde{\boldsymbol{K}}_{o I}\right) \\
& -2 \operatorname{tr}\left(\Delta \boldsymbol{K}_{o I} \boldsymbol{Q}_{K}^{-1} \tilde{\boldsymbol{K}}_{o I}\right) \\
= & -\operatorname{vec}\left(\Delta \tilde{\boldsymbol{K}}_{o I}\right)^{T} \cdot\left(\boldsymbol{I} \otimes \boldsymbol{Q}_{K}\right)^{-1} \cdot \operatorname{vec}\left(\Delta \tilde{\boldsymbol{K}}_{o I}\right) \\
& -2 \boldsymbol{s}^{T} \tilde{\boldsymbol{K}}_{o I} \Delta \boldsymbol{y}+2 \gamma \operatorname{tr}\left(\boldsymbol{K}_{o I}^{T} \tilde{\boldsymbol{K}}_{o I}\right) .
\end{aligned}
$$

Only need to perform a similar derivation for (A.2) can we obtain:

$$
\begin{aligned}
{\left[\operatorname{vec}\left(\tilde{\boldsymbol{D}}_{o I}(\tau)\right)^{T}-\operatorname{vec}\left(\tilde{\boldsymbol{D}}_{o I}(\tau-T)\right)^{T}\right] \cdot\left(\boldsymbol{I} \otimes \boldsymbol{Q}_{K}\right)^{-1} } \\
\quad \cdot\left[\operatorname{vec}\left(\tilde{\boldsymbol{D}}_{o I}(\tau)\right)+\operatorname{vec}\left(\tilde{\boldsymbol{D}}_{o I}(\tau-T)\right)\right] \\
=-\operatorname{vec}\left(\Delta \tilde{\boldsymbol{D}}_{o I}\right)^{T} \cdot\left(\boldsymbol{I} \otimes \boldsymbol{Q}_{K}\right)^{-1} \cdot \operatorname{vec}\left(\Delta \tilde{\boldsymbol{D}}_{o I}\right) \\
\quad-2 \boldsymbol{s}^{T} \tilde{\boldsymbol{D}}_{o I} \Delta \dot{\boldsymbol{y}}+2 \gamma \operatorname{tr}\left(\boldsymbol{D}_{o I}^{T} \tilde{\boldsymbol{D}}_{o I}\right) .
\end{aligned}
$$

Considering the truth that $\boldsymbol{Q}_{K}$ and $\boldsymbol{Q}_{D}$ are positive-definite matrixes yields:

$$
\begin{gathered}
\operatorname{vec}\left(\Delta \tilde{\boldsymbol{K}}_{o I}\right)^{T} \cdot\left(\boldsymbol{I} \otimes \boldsymbol{Q}_{K}\right)^{-1} \cdot \operatorname{vec}\left(\Delta \tilde{\boldsymbol{K}}_{o I}(\tau)\right) \\
\quad=\operatorname{tr}\left[\Delta \tilde{\boldsymbol{K}}_{o I}^{T} \cdot \boldsymbol{Q}_{K}^{-1} \cdot \Delta \tilde{\boldsymbol{K}}_{o I}(\tau)\right] \geq 0 \\
\operatorname{vec}\left(\Delta \boldsymbol{D}_{o I}\right)^{T} \cdot\left(\boldsymbol{I} \otimes \boldsymbol{Q}_{D}\right)^{-1} \cdot \operatorname{vec}\left(\tilde{\boldsymbol{D}}_{o I}(\tau)\right) \\
\quad=\operatorname{tr}\left[\Delta \tilde{\boldsymbol{D}}_{o I}^{T} \cdot \boldsymbol{Q}_{D}^{-1} \cdot \Delta \tilde{\boldsymbol{D}}_{o I}(\tau)\right] \geq 0 .
\end{gathered}
$$

Folding (A.5) and (A.7) into (A.1) and (A.2) respectively, one has:

$$
\begin{aligned}
& \Delta V_{a k} \leq \int_{t-T}^{t}\left[-\boldsymbol{s}^{T} \tilde{\boldsymbol{K}}_{o I} \Delta \boldsymbol{y}+\gamma \operatorname{tr}\left(\boldsymbol{K}_{o I}^{T} \tilde{\boldsymbol{K}}_{o I}\right)\right] d \tau \\
& \Delta V_{a d} \leq \int_{t-T}^{t}\left[-\boldsymbol{s}^{T} \tilde{\boldsymbol{D}}_{o I} \Delta \dot{\boldsymbol{y}}+\gamma \operatorname{tr}\left(\boldsymbol{D}_{o I}^{T} \tilde{\boldsymbol{D}}_{o I}\right)\right] d \tau .
\end{aligned}
$$

Incorporating (A.8), (A.9), (39) and (43) into (47) we can obtain:

$$
\begin{aligned}
\Delta V= & \Delta V_{3}+\Delta V_{a k}+\Delta V_{a d} \\
\leq & \int_{t-T}^{t}\left(\boldsymbol{s}^{T} \tilde{\boldsymbol{K}}_{o I} \Delta \boldsymbol{y}+\boldsymbol{s}^{T} \tilde{\boldsymbol{D}}_{o I} \Delta \dot{\boldsymbol{y}}+\boldsymbol{s}^{T} \boldsymbol{V}_{o} \boldsymbol{s}+\boldsymbol{s}^{T} \boldsymbol{M}_{o} \Lambda \Delta \dot{\boldsymbol{y}}\right) d \tau \\
& +\Delta V_{2}+\Delta V_{a k}+\Delta V_{a d} \\
\leq & \int_{t-T}^{t}\left[\gamma \operatorname{tr}\left(\boldsymbol{K}_{o l}^{T} \tilde{\boldsymbol{K}}_{o I}\right)+\gamma \operatorname{tr}\left(\boldsymbol{D}_{o I}^{T} \tilde{\boldsymbol{D}}_{o I}\right)\right. \\
& \left.+\boldsymbol{s}^{T} \boldsymbol{M}_{o} \boldsymbol{\Lambda} \Delta \dot{\boldsymbol{y}}+\boldsymbol{s}^{T} \boldsymbol{V}_{o} \boldsymbol{s}\right] d \tau .
\end{aligned}
$$

When the gain parameters are chosen to satisfy the formula:

$$
\gamma \operatorname{tr}\left(\boldsymbol{K}_{o I}^{T} \tilde{\boldsymbol{K}}_{o I}\right)+\gamma \operatorname{tr}\left(\boldsymbol{D}_{o I}^{T} \tilde{\boldsymbol{D}}_{o I}\right)+\boldsymbol{s}^{T} \boldsymbol{M}_{o} \boldsymbol{\Lambda} \Delta \dot{\boldsymbol{y}}+\boldsymbol{s}^{T} \boldsymbol{V}_{o} \boldsymbol{s} \leq 0
$$

$\Delta V$ in (A.10) can be made non-positive. Then the asymptotic stability of this adaptive controller and the convergence of the parameters are ensured.

\section{Appendix B. Introduction to the dual-arm system}

The whole system includes a standard PC for development and debugging, a central controller and a dual-arm system. The PC is installed with Matlab/Simulink, which offers an interface for researchers to operate the robotic system and develop control algorithm. Workbench is also installed to develop projects for Vxworks. The PC communicates with the central controller via Gigabit Ethernet. The central controller and the dual-arm robotic system are interconnected by ppseco which can provide a maximum data transfer rate of $25 \mathrm{Mb} / 200$ us, it is a communication protocol developed by our laboratory. The EPIC-based central controller is equipped with Intel Core Duo processor, which can provide 12575 MFLOPS floating-point arithmetic capability. An 8 GB memory card is inserted in the type-2 CompactFlash interface provided by the central controller, it is used to install the Vxworks operating system and store experimental data. Vxworks is a real-time embedded operating system running at $1 \mathrm{kHz}$. The sampling period of the position sensors integrated in the modular joint are $200 \mu \mathrm{s}$. Paul plan is adopted as the joint-space plan, which generates joint trajectory per $100 \mathrm{~ms}$. This algorithm can prevent vibration and jerk and ensure a smooth motion of the manipulators [40]. The central controller receives the real-time position/joint-torque signals sampled by each sensor and then generates motor drive signals via trajectory planning and control algorithm. 
The speed of response of the inner loop is much higher than that of outer loop, namely, the inner loop possesses higher bandwidth or tracking frequency compared with the outer loop. This contributes to the successful implementation of the following experiments and a much faster stabilization of the whole physical system.

Two 6-DOF force-torque sensors (JR3) are mounted at the end of the two manipulators respectively with separate data processing and high-speed communication. Two end-effectors are mounted on the output side of the JR3s respectively, both of them are in cylindrical shape with small diameter and made of aluminum alloy.

\section{References}

[1] S. Lee, Dual redundant arm configuration optimization with task-oriented dual arm manipulability, IEEE Trans. Robot. Autom. 5 (1) (1989) 78-97.

[2] N. Hogan, Impedance control: An approach to manipulation, in: American Control Conference, 1984, IEEE, 1984, pp. 304-313.

[3] N. Hogan, Stable execution of contact tasks using impedance control, in: 1987 IEEE International Conference on Robotics and Automation. Proceedings, Vol. 4, IEEE, 1987, pp. 1047-1054.

[4] N. Hogan, On the stability of manipulators performing contact tasks, IEEE J Robot. Autom. 4 (6) (1988) 677-686.

[5] D.E. Whitney, Historical perspective and state of the art in robot force control, Int. J. Robot. Res. 6 (1) (1987) 3-14.

[6] M. Uchiyama, P. Dauchez, Symmetric kinematic formulation and nonmaster/slave coordinated control of two-arm robots, Adv. Robot. 7 (4) (1992) 361-383.

[7] J. Lee, P.H. Chang, R.S. Jamisola, Relative impedance control for dual-arm robots performing asymmetric bimanual tasks, IEEE Trans. Ind. Electron. 61 (7) (2014) 3786-3796.

[8] W.H. Zhu, J.D. Schutter, Control of two industrial manipulators rigidly holding an egg, IEEE Control Syst. 19 (2) (1999) 24-30.

[9] R.G. Bonitz, T.C. Hsia, Internal force-based impedance control for cooperating manipulators, IEEE Trans. Robot. Autom. 12 (1) (1996) 78-89.

[10] M. Koga, K. Kosuge, K. Furuta, K. Nosaki, Coordinated motion control of robot arms based on the virtual internal model, IEEE Trans. Robot. Autom. 8 (1) (1992) 77-85

[11] T. Wimböck, C. Ott, G. Hirzinger, Impedance behaviors for two-handed manipulation: Design and experiments, in: 2007 IEEE International Conference on Robotics and Automation, IEEE, 2007, pp. 4182-4189.

[12] S. Schneider, R.H. Cannon Jr., Object impedance control for cooperative manipulation: Theory and experimental results, IEEE Trans. Robot. Autom. 8 (3) (1992) 383-394

[13] K.Y. Lian, C.S. Chiu, P. Liu, Semi-decentralized adaptive fuzzy control for cooperative multirobot systems with $\mathrm{H} \infty$ motion/internal force tracking performance, IEEE Trans. Syst. Man Cybern. B 32 (3) (2002) 269-280.

[14] W. Gueaieb, F. Karray, S. Al-Sharhan, A robust hybrid intelligent position/force control scheme for cooperative manipulators, IEEE/ASME Trans. Mechatronics 12 (2) (2007) 109-125.

[15] W. Gueaieb, F. Karray, S. Al-Sharhan, A robust adaptive fuzzy position/force control scheme for cooperative manipulators, IEEE Trans. Control Syst. Technol. 11 (4) (2003) 516-528.

[16] F. Caccavale, P. Chiacchio, A. Marino, L. Villani, Six-DOF impedance control of dual-arm cooperative manipulators, IEEE/ASME Trans. Mechatronics 13 (5) (2008) 576-586.

[17] F. Caccavale, L. Villani, Impedance control of cooperative manipulators, Mach. Intell. Robot. Control 2 (2000) 51-57.

[18] M.E. Abdallah, C.W. Wampler, R. Platt Jr., Object impedance control using a closed-chain task definition, in: 2010 10th IEEE-RAS International Conference on Humanoid Robots (Humanoids), IEEE, 2010, pp. 269-274.

[19] S.A.A. Moosavian, R. Rastegari, Multiple-arm space free-flying robots for manipulating objects with force tracking restrictions, Robot. Auton. Syst. 54 (10) (2006) 779-788.

[20] C. Smith, Y. Karayiannidis, L. Nalpantidis, X. Gratal, P. Qi, D.V. Dimarogonas, D. Kragic, Dual arm manipulation-A survey, Robot. Auton. Syst. 60 (10) (2012) $1340-1353$

[21] T. Kulvicius, M. Biehl, M.J. Aein, M. Tamosiunaite, F. Wörgötter, Interaction learning for dynamic movement primitives used in cooperative robotic tasks, Robot. Auton. Syst. 61 (12) (2013) 1450-1459.

[22] C. Yang, G. Ganesh, S. Haddadin, S. Parusel, A. Albu-Schäeffer, E. Burdet Human-like adaptation of force and impedance in stable and unstable interactions, IEEE Trans. Robot. 27 (5) (2011) 918-930

[23] J. Buchli, F. Stulp, E. Theodorou, S. Schaal, Learning variable impedance control, Int. J. Robot. Res. 30 (7) (2011) 820-833.

[24] K.P. Tee, D.W. Franklin, M. Kawato, T.E. Milner, E. Burdet, Concurrent adaptation of force and impedance in the redundant muscle system, Biol. Cybernet. 102 (1) (2010) 31-44

[25] B. Siciliano, O. Khatib (Eds.), Springer Handbook of Robotics, Springer Science \& Business Media, 2008

[26] T. Wimböck, C. Ott, A. Albu-Schäffer, G. Hirzinger, Comparison of object-level grasp controllers for dynamic dexterous manipulation, Int. J. Robot. Res. 31 (1) (2012) 3-23.
[27] F. Caccavale, L. Villani, An impedance control strategy for cooperative manipulation, in: 2001 IEEE/ASME International Conference on Advanced Intelligent Mechatronics, 2001. Proceedings, Vol. 1, IEEE, 2001, pp. 343-348.

[28] A. Prochazka, P.S. Trend, Instability in human forearm movements studied with feedbackcontrolled muscle vibration, J. Physiol. 402 (1) (1988) 421-442.

[29] J. Won, N. Hogan, Stability properties of human reaching movements, Exp. Brain Res. 107 (1) (1995) 125-136.

[30] I.D. Walker, R.A. Freeman, S.I. Marcus, Analysis of motion and internal loading of objects grasped by multiple cooperating manipulators, Int. J. Robot. Res. 10 (4) (1991) 396-409.

[31] K.L. Doty, C. Melchiorri, C. Bonivento, A theory of generalized inverses applied to robotics, Int. J. Robot. Res. 12 (1) (1993) 1-19.

[32] J.K. Salisbury, Active stiffness control of a manipulator in Cartesian coordinates, in: 1980 19th IEEE Conference on Decision and Control Including the Symposium on Adaptive Processes, IEEE, 1980, pp. 95-100.

[33] F. Caccavale, S. Chiaverini, B. Siciliano, Second-order kinematic control of robot manipulators with Jacobian damped least-squares inverse: Theory and experiments, IEEE/ASME Trans. Mechatronics 2 (3) (1997) 188-194.

[34] J. Wang, Y. Li, X. Zhao, Inverse kinematics and control of a 7-dof redundant manipulator based on the closed-loop algorithm, Int. J. Adv. Rob. Syst. 7 (4) (2010) 1-9.

[35] M.A. Unseren, A rigid body model and decoupled control architecture for two manipulators holding a complex object, Robot. Auton. Syst. 10 (2) (1992) $115-131$

[36] H.K. Khalil, Adaptive output feedback control of nonlinear systems represented by input-output models, IEEE Trans. Autom. Control 41 (2) (1996) $177-188$.

[37] D. Heck, D. Kostic, A. Denasi, H. Nijmeijer, Internal and external forcebased impedance control for cooperative manipulation, in: Control Conference (ECC), 2013 European, IEEE, 2013, pp. 2299-2304.

[38] H. Bai, J.T. Wen, Cooperative load transport: a formation-control perspective, IEEE Trans. Robot. 26 (4) (2010) 742-750.

[39] D. Sun, Y. Liu, J.K. Mills, Cooperative control of a two-manipulator system handling a general flexible object, in: Proceedings of the 1997 IEEE/RS] International Conference on Intelligent Robots and Systems, 1997. IROS'97. Vol. 1, IEEE, 1997, pp. 5-10.

[40] R.P. Paul, Robot Manipulators: Mathematics, Programming, and Control: The Computer Control of Robot Manipulators, Richard Paul, 1981.

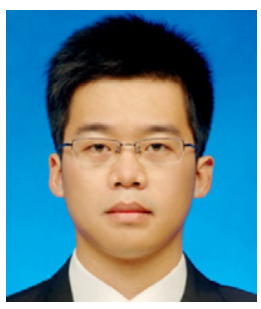

Yi Ren was born in 1989. He is currently a Ph.D. candidate in State Key Laboratory of Robotics and System at Harbin Institute of Technology (HIT) in China. He received his B.S. (2011) in Thermal Energy and Power Engineering and M.S. (2013) in Mechatronics Engineering at HIT. His research interests include robotics, control of redundant manipulator/dual-arm cooperation and object grasping.

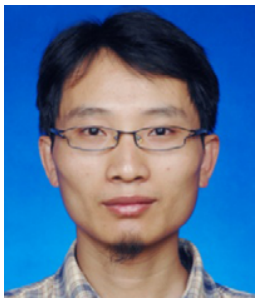

Yechao Liu received his B.S. (2002) in Thermal Energy and Power Engineering and M.S. (2004) in Mechatronics Engineering from Harbin Institute of Technology (HIT), Harbin, PRC. Now he is an assistant professor at the school of Mechanic Engineering in HIT. His research interests are in the area of robotics, dynamics algorithms of flexiblejoint manipulators and their compliance control.

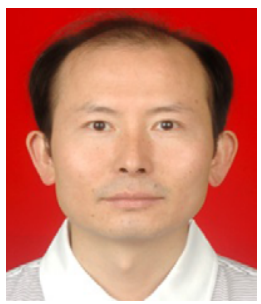

Minghe Jin received his B.S. degree in Vehicle Engineering in 1993, and the M.S. and Ph.D. degrees in Mechanical Engineering, in 1996 and 2000, respectively, all from Harbin Institute of Technology (HIT), Harbin, PRC. Now he is a professor in Robotics Institute in HIT. His research interests include dexterous robot hands, space robotics and real-time embedded control system.

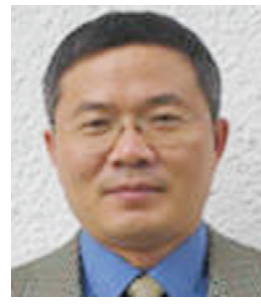

Hong Liu received the Ph.D. degree in 1993 from Harbin Institute of Technology, PRC. His doctoral research was in the area of macro-micro manipulator systems. During 1991-1993 he worked as a joint-Ph.D. candidate in the Institute of Robotics and System Dynamics at the German Aerospace Research Establishment in Germany. Since 1993 he has been a Research Fellow in this institute. He is currently a professor in HIT. His research projects include the development of a dexterous robot hand, space manipulator, etc. 